P. M. A. Areias · J. M. A. César de Sá

C. A. Conceição António - J. A. S. A. O. Carneiro

V .M. P. Teixeira

\title{
Strong displacement discontinuities and Lagrange multipliers in the analysis of finite displacement fracture problems
}

Received: 19 April 2004 / Accepted: 25 May 2004 / Published online: 18 August 2004

(C) Springer-Verlag 2004

\begin{abstract}
The finite displacement formulation of a quadrilateral element containing an embedded displacement discontinuity is presented. The formulation is based on the kinematically optimal technique, and some known defects of this technique are addressed. Lagrange multipliers are adopted to ensure the correct crack closure prior to initiation. Six additional degrees of freedom in each element allow the representation of the two states of the crack. A classical enhanced strain technique is employed to improve the bending performance of the element. Numerical examples illustrate both the robustness and the accuracy of the proposed solutions.
\end{abstract}

Keywords Embedded discontinuities - Finite displacement · Quadrilateral element · Lagrange multipliers - KOS formulation · Enhanced strain technique

\section{General considerations}

The finite element analysis of brittle crack initiation and propagation in solids can be carried out through the use of embedded strong displacement discontinuities. The basic developments of this technique are supported by both the accurate representation of the displacement field induced by the presence of a crack and the stress continuity condition between the crack faces and the continuum $[1,2,3,4]$.

The main attractiveness of this technique is the possibility of crack propagation along arbitrary paths

P. M. A. Areias $(\bowtie)$ · J. M. A. César de Sá

C. A. Conceição António

Faculdade de Engenharia, Universidade do Porto, 4200-465 Porto, Portugal

J. A. S. A. O. Carneiro · V .M. P. Teixeira

Departamento de Fsica da,

Universidade do Minho, Portugal without remeshing. This relative generality is obtained through the incorporation, in each finite element, of the discontinuous displacement field caused by the presence of a crack (a review of the particular procedures to accomplish this incorporation was carried out by Jirasek [5] in 2000).

Studies concerning embedded displacement discontinuities are presented in various important references [2, 6-11] for small strain situations and in some references [12-14] for finite strain situations.

The introduction of a displacement discontinuity can also be achieved by the extended finite element technique (see [15-17]). Some of its advantages are the higher decoupling between the underlying discretization and the crack path and the exceptional accuracy in the representation of near crack tip fields, due to a special enrichment of the crack tip (e.g. [15]). However it involves the costs of a non-standard numerical quadrature procedure and also a somehow non-trivial implementation. If a given study is focused on obtaining a reasonable crack path and accurate load-displacement curve, it is clear that embedded displacement discontinuities are still attractive.

Regarding the particular formulation, three families elements with embedded displacement discontinuities $[5,18]$ are known to exist:

- SOS - symmetric statically optimal formulation

- KOS - symmetric kinematically optimal formulation

- SKON - non-symmetric statically and kinematically optimal formulation

One of the alternatives to these discrete approaches is the so called local approach of fracture [19-22]. Notwithstanding its appeal, as it circumvents the need for explicit crack discretization, the constitutive equations have to be regularized to avoid spurious mesh size dependency [23, 24]. This regularization demands a length scale parameter which is seldom easily identifiable and frequently induces too wide softening zones $[20,23]$. However, an important advantage of a local approach based on a scalar damage variable is that all 
stress components are forced to become zero with increasingly higher values of the damage variable, and hence stress locking does not occur as in standard symmetric formulations (SOS and KOS) of embedded discontinuity elements (specially in the SOS case).

From the three formulations listed above, the symmetric ones are attractive both because they are variationally consistent, giving rise to a symmetric tangent stiffness matrix, and possess a relatively straightforward formulation. Of the two symmetric formulations (KOS and SOS), the most attractive one from the formal point of view appears to be KOS. Being based on a displacement-consistent enhanced strain displacement operator, it circumvents the requirement of zero mean value, as it is the case of the EAS (enhanced assumed strain) based SOS. This allows the possibility of easily incorporating complex geometrical enhancements in the element.

Apparently, as late as 2000 [5], the only formulation based on KOS was the one of Lofty and Shing [25]. A substantial reason for this lack of popularity is that, according to the thorough study by Jirasek [5] focusing on triangular elements, "...the natural traction continuity can be severely violated". A direct consequence of this fact is that spurious tractions may appear for meshes not aligned with the crack path. In the case of finite displacement analyses, the deformation usually modifies the cracked elements' geometry and therefore induces locking even for initially aligned meshes.

This deficiency is verified and addressed in the present work. As a remedy, a modification of the constitutive stress tensor is proposed, which effectively removes the stress jump effect by the introduction of a cohesiveconsistent stress tensor.

Some differentiating characteristics of the proposed formulation are:

- A variationally consistent kinematically optimal symmetric (KOS) formulation, in the sense discussed in [5], but generalized for the finite strain case

- The consideration of a symmetric crack opening including rotation, giving rise to an additive decomposition of the deformation gradient, instead of a multiplicative one (as in Oliver et al. [14])

- Adoption of a standard Gaussian quadrature scheme for the continuum, instead of a mixed quadrature required to enforce the traction continuity condition as presented in reference [14]. Comparatively complex quadrature schemes are required in the extended finite element method [16]

- Absence of penalty parameters or active set strategies for crack closure prior to localization (which are currently widely spread)

- Stress-locking free behavior with a symmetric formulation (which usually gives rise to stress locking, or, in some cases, slow convergence of the results as discussed in reference [18])
- Coupled enhanced strain method for improving the element performance

Accordingly, some of the previously existent obstacles for a wider acceptance of KOS formulation are addressed with some level of success.

Two other aspects for the efficient implicit analysis of crack propagation are algorithmic robustness and the verified convergence behavior of the Newton-Raphson method.

Therefore, the exact linearization of the final discretized equilibrium equations is carried out.

Examples illustrating both the accuracy and robustness of the proposed solutions are exposed. Other successfully accomplished examples (with a simplified formulation) are contained in the proceedings of a recent fracture mechanics conference [26].

\section{The kinematics of the element}

\subsection{Notation}

For conciseness, general considerations regarding the presence of a displacement discontinuity can be consulted elsewhere (e.g. [3, 10, 12, 13, 17, 27]).

Relative to the kinematics, a single quadrilateral element with an embedded displacement discontinuity is studied. Both the crack mid-line and the crack faces remain straight during propagation. One crack is considered in each element, see Fig. 1.

The crack mid-line is identified through the vector $\boldsymbol{O P}$ and the crack position is identified by the position of the point $O$.

Two local director vectors corresponding to a local frame are also represented, which can be obtained from the vector $\boldsymbol{O P}: \boldsymbol{S}$ is the tangential vector and $\boldsymbol{N}$ is the normal vector. The normal vector is calculated from the stress state, as discussed in Sect. 4.1.

The proposed low order element with embedded discontinuity contains 4 nodes $(k=1,2,3,4)$, as illustrated in Fig. 1. This figure also presents three sets of points, $\Omega_{1}$ and $\Omega_{2}$ (such as $\Omega_{1} \cap \Omega_{2}=\emptyset$ ) and $\Gamma$. This last set of points represents the crack.

The complete element as a set of points is identified as $\Omega$, or $\Omega=\Omega_{1} \cup \Omega_{2} \cup \Gamma$ with $\Omega_{1} \cap \Gamma=\emptyset$ and $\Omega_{2} \cap \Gamma=\emptyset$.

In the presently adopted notation, a given point is denoted as $X$, its material and spatial positions are denoted as $\boldsymbol{X}$ and $\boldsymbol{x}$ respectively. A converged position of $X$ at the instant of crack initiation is identified as $\boldsymbol{X}^{*}$. Finally, the $i^{\text {th }}$ scalar component of $\boldsymbol{X}$ is represented as $X_{i}$.

Before proceeding, it may be helpful to think of starred quantities such as $\boldsymbol{X}^{*}$ as belonging to an intermediate incompatible configuration which is defined for each element independently and corresponds to the instant of crack initiation in that particular element. 
Fig. 1 The illustration of the embedded discontinuity for a single quadrilateral element (OP represents the crack mid-line). A configuration with crack opening is shown

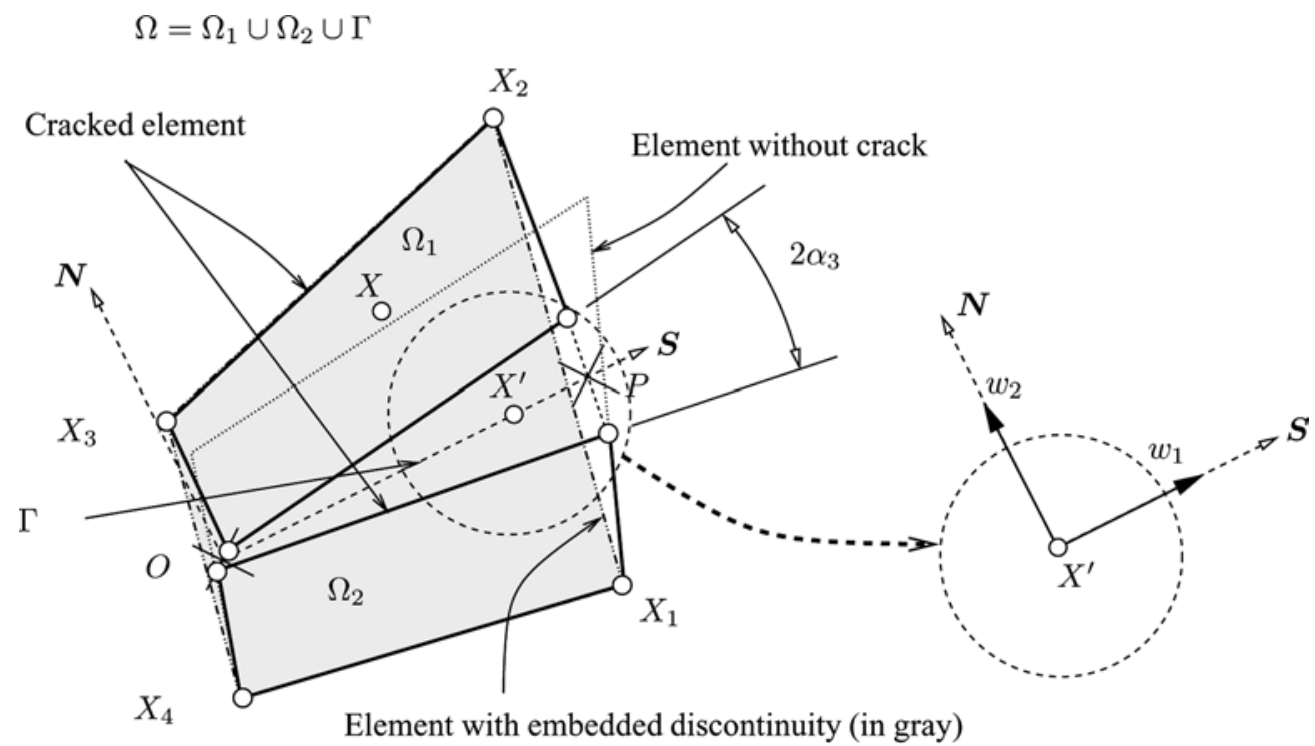

2.2 Rigid body displacement field resultant from the crack

The existence of a crack, $\Gamma$, which separates the element in two parts, $\Omega_{1}$ and $\Omega_{2}$ (see Fig. 1) allows rigid body motions in these two parts.

The rigid body displacement field of the two element parts should be considered in addition to the original displacement field corresponding to the non-cracked element.

If the converged coordinates $\boldsymbol{X}^{*}$ of an arbitrary point $X \in \Omega_{1} \cup \Omega_{2}$ are considered, the displacement vector of this point due to the crack induced rigid body motion can be calculated as:

$$
\begin{aligned}
\overline{\boldsymbol{u}}\left(\boldsymbol{X}^{*}, r\right) & =\left[\begin{array}{ll}
S_{1}^{*} & N_{1}^{*} \\
S_{2}^{*} & N_{2}^{*}
\end{array}\right] \\
& \times\left\{\begin{array}{l}
\boldsymbol{O}^{*} \boldsymbol{X}^{*} \cdot \boldsymbol{S}^{*}\left(\cos \alpha_{3}-1\right)-r \boldsymbol{O}^{*} \boldsymbol{X}^{*} \cdot \boldsymbol{N}^{*} \sin \alpha_{3}+r \alpha_{1} \\
r \boldsymbol{O}^{*} \boldsymbol{X}^{*} \cdot \boldsymbol{S}^{*} \sin \alpha_{3}+\boldsymbol{O}^{*} \boldsymbol{X}^{*} \cdot \boldsymbol{N}^{*}\left(\cos \alpha_{3}-1\right)+r \alpha_{2}
\end{array}\right\}
\end{aligned}
$$

where $\alpha_{1}, \alpha_{2}, \alpha_{3}$ are kinematic degrees of freedom and $r$ is a number belonging to the set $\{-1,1\}$.

The variable $\alpha_{3}$ represents the crack face rotation. The variables $\alpha_{1}$ and $\alpha_{2}$ represent the local displacements components of the upper crack face at point $O$ (see Fig. $1)$. The notation $\alpha_{i}$ agrees with the convention adopted for EAS elements, as exposed in references [28-30].

These degrees of freedom $\alpha_{i}$ are additive, in contrast with the result $\overline{\boldsymbol{u}}$. A representation of the scalar components of $\overline{\boldsymbol{u}}$ in (1) can be written using the notation $\bar{u}_{1}=f_{1}\left(\alpha_{i}\right)$ and $\bar{u}_{2}=f_{2}\left(\alpha_{i}\right)$ with $i=1, \ldots, 3$. The functions $f_{1}$ and $f_{2}$ are introduced to allow the representation of the displacement jump at $\Gamma$.

It is noted that both the point $O$ and the three internal variables $\alpha_{i}$ are sufficient to describe the crack-induced rigid body displacement field in $\Omega_{1}$ and $\Omega_{2}$.

The motivation behind use of the parameter $r \in\{-1,1\}$ in Eq (1) is to simply identify the set of points to which $X$ belongs. If $X \in \Omega_{1}$ then $r=1$ and if $X \in \Omega_{2}$ then $r=-1$.

For the analysis of the crack opening, it is necessary to rewrite a relation analogous to (1) in local coordinates and valid for $X^{\prime} \in \Gamma$ as depicted in Fig. 1 .

In the local frame, the scalar components of the relative displacement at $\Gamma$ can be evaluated as:

$w_{1}=2 \alpha_{1} \quad$ in $\Gamma$

$w_{2}=2\left\|\boldsymbol{O}^{*} \boldsymbol{X}^{x^{\prime}}\right\| \sin \alpha_{3}+2 \alpha_{2} \quad$ in $\Gamma$

with $X^{\prime} \in \Gamma$ (see Fig. 1).

The relations (2) represent local coordinates of the displacement jump at the crack.

To guarantee that no penetration takes place between $\Omega_{1}$ and $\Omega_{2}$ it is necessary to verify the condition $w_{2} \in \Re_{0}^{+}$.

The global displacement $\overline{\boldsymbol{w}}$ corresponding to the local coordinates $w_{1}$ and $w_{2}$ can be written as:

$\overline{\boldsymbol{w}}=\left[\begin{array}{ll}S_{1}^{*} & N_{1}^{*} \\ S_{2}^{*} & N_{2}^{*}\end{array}\right]\left\{\begin{array}{l}w_{1} \\ w_{2}\end{array}\right\} \quad$ in $\Gamma$

If the crack is closed, both $\overline{\boldsymbol{u}}$ and $\overline{\boldsymbol{w}}$ should be null vectors, a condition that can be trivially satisfied by ensuring that $\alpha_{i}=0, i=1,2,3$.

The relation (1) can be specified for a given node $k$, according to:

$\overline{\boldsymbol{u}}_{k}=\overline{\boldsymbol{u}}\left[\boldsymbol{X}_{k}^{*}, \frac{\left(\boldsymbol{X}_{k}^{*}-\boldsymbol{O}^{*}\right) \cdot \boldsymbol{N}^{*}}{\left|\left(\boldsymbol{X}_{k}^{*}-\boldsymbol{O}^{*}\right) \cdot \boldsymbol{N}^{*}\right|}\right]$

in $\Omega_{1} \cup \Omega_{2}$. This particular case consists on a replacement of $\boldsymbol{X}^{*}$ in Eq. (1) by $\boldsymbol{X}_{k}^{*}$ and the introduction of the value $r$ which depends on the relative node position.

A notation for the scalar components of $\overline{\boldsymbol{u}}_{k}$ in (4) can be carried out as: $\bar{u}_{k 1}=f_{k 1}\left(\alpha_{1}, \alpha_{2}, \alpha_{3}\right)$ and $\bar{u}_{k 2}=f_{k 2}$ $\left(\alpha_{1}, \alpha_{2}, \alpha_{3}\right)$.

With the introduction of the particular case (4), it is then possible to define the contribution of the crack to the rigid body displacement field as: 
$\overline{\boldsymbol{u}}=H_{k} \overline{\boldsymbol{u}}_{k}$

where $H_{k}$ are the standard isoparametric shape functions for the quadrilateral. This type of isoparametric representation of the rigid body kinematics has been considered for triangular elements in references [4,13,27], but without enforcing the symmetry of the crack mid-line $O P$.

The total displacement field, $\boldsymbol{u}$, in $\Omega_{1} \cup \Omega_{2}$ can then be determined as the difference between the regular displacement (which is identified with a hat, $\widehat{\boldsymbol{u}}$ ) and the jump displacement:

$\boldsymbol{u}=\underbrace{\widehat{\boldsymbol{u}}}_{\text {Regular }}-\underbrace{\overline{\boldsymbol{u}}}_{\text {Jump }}$ in $\Omega_{1} \cup \Omega_{2}$

The regular displacement field corresponds to the displacement that occurs in the element represented in gray in Fig. 1, but without considering the discontinuity.

The isoparametric interpolation concerning the displacement field, $\boldsymbol{u}$, is established as:

$\boldsymbol{u}=\underbrace{H_{k} \boldsymbol{u}_{k}}_{\text {Nodal regular }}-\underbrace{H_{k} \overline{\boldsymbol{u}}_{k}}_{\text {Nodal jump }} \quad$ in $\Omega_{1} \cup \Omega_{2}$

with $H_{k}=0.25\left(1+\xi_{1 k} \xi_{1}\right)\left(1+\xi_{2 k} \xi_{2}\right)$ where $\xi_{1}$ and $\xi_{2}$ are local isoparametric coordinates. Accordingly, $\xi_{1 k}$ and $\xi_{2 k}$ represent the values of the coordinates $\xi_{1}$ and $\xi_{2}$ at the given nodes $k$.

It is clear that both Eqs. (6) and (7) are written for $\Omega_{1} \cup \Omega_{2}$ only. Hence, they are generally invalid in $\Omega$. Another observation is that $\overline{\boldsymbol{u}}_{k}$ in (7) does not represent a nodal variable, but rather the enhanced displacement vector evaluated in nodes $k$.

Along the same lines, the discretized displacement field in (7) is smooth, as finite strain values exist in $\Omega_{1} \cup \Omega_{2}$ (likewise, the introduction of a displacement shift, as proposed in ref [18], does not affect the format of the equilibrium equations).

2.3 The deformation gradient and the spatial velocity gradient

With the definition of the displacement increment field, and hence of the displacement field, the derived quantities follow using standard relations from continuum mechanics (see the presentation in reference [31]).

The discretized form of the deformation gradient can be written as:

$\boldsymbol{F}=\widehat{\boldsymbol{F}}-\overline{\boldsymbol{F}}+\boldsymbol{I}+\delta_{\Gamma} \overline{\boldsymbol{w}} \otimes \boldsymbol{N} \quad$ in $\Omega$

As the Eq. (8) is defined in $\Omega$, it includes the effect of the discontinuous displacement field, and hence of the unbounded strain along the crack path $\Gamma$.

The term $\delta_{\Gamma}$ in Eq. (8) represents the Dirac delta function defined on the set $\Gamma$. The terms $\widehat{\boldsymbol{F}}$ and $\overline{\boldsymbol{F}}$ can be evaluated according to their scalar components:

$\widehat{F}_{i j}=\frac{\partial H_{k}}{\partial X_{j}}\left(X_{k i}+u_{k i}\right) \quad$ in $\Omega_{1} \cup \Omega_{2}$ and

$\bar{F}_{i j}=\frac{\partial H_{k}}{\partial X_{j}}\left(X_{k i}+\bar{u}_{k i}\right) \quad$ in $\Omega_{1} \cup \Omega_{2}$

The spatial velocity gradient (in $\Omega$ ) is usually denoted as $\boldsymbol{L}$ with its scalar components written as:

$L_{i j}=\frac{\partial H_{k}}{\partial x_{j}} \dot{u}_{k i}+g_{i j p} \dot{\alpha}_{p}+\delta_{\Gamma} \dot{\bar{w}}_{i} N_{k} F_{k j}^{-1} \quad$ in $\Omega$

where use was made of the following relation:

$\frac{\partial H_{r}}{\partial X_{s}}=\frac{\partial H_{r}}{\partial x_{p}} F_{p s}$

In Eq. (11), the quantity

$g_{i j p}=-\frac{\partial H_{r}}{\partial X_{k}} \frac{\partial X_{k}}{\partial x_{j}} \frac{\partial f_{r i}}{\partial \alpha_{p}}$

was introduced.

The improvement of the deformation gradient field introduced by the Eq. (8) is carried out using the standard enhanced assumed strain (EAS) technique based on the bubble function $[30,32]$. Therefore, a modified deformation gradient, $\boldsymbol{F}$, is introduced as:

$\widetilde{\boldsymbol{F}}=\widehat{\boldsymbol{F}}-\overline{\boldsymbol{F}}+\boldsymbol{I}+\delta_{\Gamma} \overline{\boldsymbol{w}} \otimes \boldsymbol{N}+\boldsymbol{E}(\boldsymbol{\beta}) \quad$ in $\Omega$

where $\boldsymbol{\beta}$ are additional degrees of freedom. The scalar components of $\boldsymbol{E}$ in (14) can be written as:

$E_{i j}=\frac{\operatorname{det}\left[\frac{\overline{\partial X}}{\partial \xi}\right]}{\operatorname{det}\left[\frac{\partial \boldsymbol{X}}{\partial \xi}\right]} \frac{\partial B}{\partial \xi_{k}} \overline{\left(\frac{\partial \xi_{k}}{\partial X_{j}}\right)} \beta_{k i}, \quad k=1,2 \quad$ and $\quad \mathrm{i}=1,2$

with $B$ being the bubble function: $B=\left(1-\xi_{1}^{2}\right)\left(1-\xi_{2}^{2}\right)$ and $\left(\frac{\partial \xi_{k}}{\partial X_{j}}\right)$ the material average of the Jacobian matrix inverse (see also reference [30]). The over-bar notation in (15) indicates material averages of the corresponding quantities.

Motivations for the use of the function $B$ are exposed in references $[30,32]$.

Using Eq. (14), the particular form for the scalar components of the spatial velocity gradient is:

$\widetilde{L}_{i j}=L_{i j}+\frac{\partial B}{\partial \xi_{k}} \overline{\left(\frac{\partial \xi_{k}}{\partial X_{l}}\right)} \frac{\partial X_{l}}{\partial x_{j}} \frac{\operatorname{det}\left[\frac{\overline{\partial \boldsymbol{X}}}{\partial \xi}\right]}{\operatorname{det}\left[\frac{\partial \boldsymbol{X}]}{\partial \xi}\right]} \dot{\beta}_{k i}$

Finally, the spatial deformation rate can be calculated according to its definition:

$\widetilde{\boldsymbol{D}}=\frac{\widetilde{\boldsymbol{L}}^{T}+\widetilde{\boldsymbol{L}}}{2}$

2.4 Existence of the displacement discontinuity and crack closure

When the displacement discontinuity is not present, before the crack initiation, then $\alpha_{i}=0$. This condition is imposed by the introduction of a function $h$ of the crack state, defined as follows: 
$h= \begin{cases}0 & \text { crack closed } \\ 1 & \text { crack opened }\end{cases}$

If $h=0$ then $\alpha_{i}=0$ for $i=1,2,3$. A method for imposing the constraints $\alpha_{i}=0$ is the Lagrange multiplier method, which is here adopted.

Regarding the crack closure, it is clear that the displacement discontinuity, which is characterized through the variables $\alpha_{1}, \alpha_{2}, \alpha_{3}$, cannot be arbitrary, in the sense that penetration between $\Omega_{1}$ and $\Omega_{2}$ should be prevented. A statement of this constraint can be exposed as:

$l \sin \alpha_{3}+\alpha_{2} \geq 0$

$\alpha_{2} \geq 0$

where $l$ is the length of the crack inside the considered element.

The constraint is only meaningful after crack initiation. Therefore, instead of being explicitly introduced, the conditions (18) are incorporated in the discrete crack compliance law.

These conditions (18) motivate the use of a nodal quadrature for the equilibrium equations at the crack.

In other words care is taken to ensure that $\Gamma \cap\left(\partial \Omega_{1} \cup \partial \Omega_{2}\right)$ is a set containing two points.

\section{Equilibrium equations and related discretized forms}

Let $V_{0}$ represent the material integration volume corresponding to the domain $\Omega_{1} \cup \Omega_{2}$ and $v_{0}$ represent the spatial integration volume corresponding to the same domain. The element is identified by $\Omega$ and its boundary by $\partial \Omega$.

Distinct finite deformation derivations in the context of embedded discontinuities have been presented in references $[12,14]$. An alternative approach is given in reference [13].

The crack zone itself has a material integration line $l_{0}$. A given point $X \in \Omega_{1} \cup \Omega_{2}$ is represented through its material coordinates $\boldsymbol{X}$ or its spatial coordinates $\boldsymbol{x}=\boldsymbol{X}+\boldsymbol{u}$. It is true that spatial positions of points belonging to one of the crack faces do not have a single image in $\Gamma$ if the crack is open, and therefore points in $\Gamma$ can only be identified by its starred or material coordinates and the crack face indication.

As $\overline{\boldsymbol{u}}(\boldsymbol{X})$ and $\widehat{\boldsymbol{u}}(\boldsymbol{X})$ are two independent displacement fields, they can be used to introduce kinematically admissible virtual displacements $\delta \overline{\boldsymbol{u}}$ and $\delta \widehat{\boldsymbol{u}}$ to project the equilibrium equations and obtain a convenient weak form.

The existence of a volume force field represented by a related material vector $\boldsymbol{b}(\boldsymbol{X})$ is postulated. This representation includes surface forces if a Dirac delta function is introduced ${ }^{1}$. Further details relative to the strong form of equilibrium in a body containing a discontinuity

${ }^{1}$ due to the fact that $\Omega \cap \partial \Omega=\emptyset$ holds, the task can be accomplished by defining forces in $\Omega$ as close as possible to $\partial \Omega$ are exposed in reference [31]. For conciseness, the relevant considerations regarding the modified spatial velocity gradient (16) are carried out after the nonmodified equilibrium equations.

If $\tau(\boldsymbol{F})$ represents the Kirchhoff stress tensor, the weak form of equilibrium can be exposed as:

$$
\begin{gathered}
\int_{V_{0}} \boldsymbol{\tau}(\boldsymbol{F}): \nabla_{\boldsymbol{x}} \delta \boldsymbol{u} \mathrm{d} V_{0}+\int_{l_{0}} \delta \overline{\boldsymbol{w}} \cdot \boldsymbol{t} \mathrm{d} l_{0} \\
=\int_{V_{0}} \boldsymbol{b} \cdot \delta \boldsymbol{u} \mathrm{d} V_{0} \quad \text { in } \Omega
\end{gathered}
$$

with $\boldsymbol{t}=\boldsymbol{\tau} \boldsymbol{F}^{-T} \boldsymbol{N}$ if the crack is closed $(h=0)$. The Kirchhoff stress tensor is obtained as a function of the Cauchy stress tensor, $\boldsymbol{\sigma}$, with the well known relation $\tau=\operatorname{det}[\boldsymbol{F}] \boldsymbol{\sigma}$.

This weak form is very similar to the standard projected form for a non-cracked element (with the noticeable exception of the crack term). It is a simple matter to separate the virtual displacement $\delta \boldsymbol{u}$ in two terms as $\delta \boldsymbol{u}=\delta \widehat{\boldsymbol{u}}+\delta \overline{\boldsymbol{u}}$ and therefore decouple the virtual work equation (19).

Introducing the Kirchhoff stress vector in the frame $\{\boldsymbol{s}, \boldsymbol{n}\}$ as $\left\{t_{1}, t_{2}\right\}^{T}$ then the equation (19) can be written as:

$$
\begin{aligned}
& \int_{V_{0}} \tau(\boldsymbol{F}): \nabla_{\boldsymbol{x}} \delta \boldsymbol{u} \mathrm{d} V_{0}+\int_{l_{0}} t_{i} \delta w_{i} \mathrm{~d} l_{0} \\
& =\int_{V_{0}} \boldsymbol{b} \cdot \delta \boldsymbol{u} \mathrm{d} V_{0} \quad \text { in } \Omega
\end{aligned}
$$

Eq. (20) can be rewritten using the scalar components of the involved quantities:

$$
\begin{gathered}
\int_{V_{0}} \tau_{i j}\left(\frac{\partial \delta \widehat{u}_{i}}{\partial x_{j}}+\frac{\partial \delta \bar{u}_{i}}{\partial x_{j}}\right) \mathrm{d} V_{0}+\int_{l_{0}} t_{i} \delta w_{i} \mathrm{~d} l_{0} \\
=\int_{V_{0}} b_{i}\left(\delta \widehat{u}_{i}+\delta \bar{u}_{i}\right) \mathrm{d} V_{0} \quad \text { in } \Omega
\end{gathered}
$$

The condition for the non-existence of the crack is satisfied imposing $\bar{u}_{i}=0$ for $h=0$.

If Lagrange multipliers $\lambda_{i}$ are introduced, then it follows that:

$$
\begin{aligned}
& \underbrace{\int_{i j}\left(\frac{\partial \delta \widehat{u}_{i}}{\partial x_{j}}+\frac{\partial \delta \bar{u}_{i}}{\partial x_{j}}\right) \mathrm{d} V_{0}+\int_{l_{0}} t_{i} \delta w_{i} \mathrm{~d} l_{0}}_{\delta V_{0}} \\
& +\underbrace{\delta \lambda_{i}\left[\alpha_{i}(1-h)+\lambda_{i} h\right]+\delta \alpha_{i} \lambda_{i}(1-h)}_{\delta \Psi} \\
& =\int_{V_{0}} b_{i} \delta u_{i} \mathrm{~d} V_{0} \quad \text { in } \Omega
\end{aligned}
$$

It is now important to acknowledge that the introduction of the three multipliers $\lambda_{i}$ as they appear in Eq. (22) is an effective way of simplifying the practical coding details.

For the application of the Newton-Raphson method, the first variations of the terms $\delta \Pi$ and $\delta \Psi$ in Eq. (21) are required. It is a straightforward matter to obtain the following equation for $\mathrm{d} \delta \Pi$ : 


$$
\begin{aligned}
\mathrm{d} \delta \Pi= & \int_{V_{0}} C_{i j k l}^{\tau} \frac{\partial \mathrm{d} u_{k}}{\partial x_{l}} \frac{\partial \delta u_{i}}{\partial x_{j}} \mathrm{~d} V_{0} \\
& +\int_{V_{0}} \tau_{k j} \frac{\partial \mathrm{d} u_{i}}{\partial x_{k}} \frac{\partial \delta u_{i}}{\partial x_{j}} \mathrm{~d} V_{0} \\
& +\int_{l_{0}} \delta w_{i} C_{i j}^{I} \mathrm{~d} w_{j} \mathrm{~d} l_{0}+\int_{l_{0}} \delta \mathrm{d} w_{i} t_{i} \mathrm{~d} l_{0} \quad \text { in } \Omega
\end{aligned}
$$

where $C_{i j k l}^{\tau}$ are the scalar components of the spatial elastic modulus such as:

$\mathscr{L}_{v} \boldsymbol{\tau}=\boldsymbol{C}^{\tau}: \boldsymbol{D}=\dot{\boldsymbol{\tau}}-\boldsymbol{\tau} \boldsymbol{L}^{T}-\boldsymbol{L} \boldsymbol{\tau}$

with $\mathscr{L}_{v} \tau$ representing the spatial velocity Lie derivative of the Kirchhoff stress tensor. This objective rate of Kirchhoff stress is convenient for the particular continuum constitutive law in use.

The notation $C_{i j}^{I}$ in (23) represents the relation between the rate of the crack surface stress (the superscript $I$ indicates local coordinates) $\dot{\boldsymbol{t}}$, and the rate of crack opening $\dot{\boldsymbol{w}}$, such as $\dot{t}_{i}=C_{i j}^{I} \dot{w}_{j}$. These coefficients will be introduced in Sect. 4.3.

Regarding $\mathrm{d} \delta \Psi$, the following equation is obtained:

$$
\begin{aligned}
\mathrm{d} \delta \Psi & =\mathrm{d} \alpha_{i} \delta \lambda_{i}(1-h)+\delta \alpha_{i} \mathrm{~d} \lambda_{i}(1-h) \\
& +h \delta \lambda_{i} \mathrm{~d} \lambda_{i}
\end{aligned}
$$

The Eqs. (22), (23) and (25) are important for the actual finite element implementation.

The discretization of Eq. (22) can be carried out as:

$$
\begin{aligned}
& \underbrace{\int_{V_{0}} \tau_{i j}\left(\frac{\partial H_{k}}{\partial x_{j}} \delta u_{k i}+g_{i j p} \delta \alpha_{p}\right) \mathrm{d} V_{0}+\int_{l_{0}} \frac{\partial w_{i}}{\partial \alpha_{p}} t_{i} \delta \alpha_{p} \mathrm{~d} l_{0}}_{\delta \Pi\left(\delta u_{k i}, \delta \alpha_{p}\right)} \\
& \underbrace{\delta \lambda_{i}\left[\alpha_{i}(1-h)+\lambda_{i} h\right]+\delta \alpha_{i} \lambda_{i}(1-h)}_{\delta \Psi\left(\delta \alpha_{i}, \delta \lambda_{i}\right)} \\
& =\int_{V_{0}} b_{i} \delta u_{i} \mathrm{~d} V_{0} \quad \text { in } \Omega
\end{aligned}
$$

For Eq. (23), it is possible to write:

$$
\begin{aligned}
& \mathrm{d} \delta \Pi\left(\delta \boldsymbol{u}_{k}, \delta \boldsymbol{\alpha}, \mathrm{d} \boldsymbol{u}_{k}, \mathrm{~d} \boldsymbol{\alpha}\right)= \\
& \int_{V_{0}} C_{i j k l}^{\tau}\left(\frac{\partial H_{p}}{\partial x_{l}} \mathrm{~d} u_{p k}+g_{k l p} \mathrm{~d} \alpha_{p}\right)\left(\frac{\partial H_{q}}{\partial x_{j}} \delta u_{q i}+g_{i j q} \delta \alpha_{q}\right) \mathrm{d} V_{0} \\
& +\int_{V_{0}} \tau_{k j}\left(\frac{\partial H_{p}}{\partial x_{k}} \mathrm{~d} u_{p i}+g_{i k p} \mathrm{~d} \alpha_{p}\right)\left(\frac{\partial H_{q}}{\partial x_{j}} \delta u_{q i}+g_{i j q} \delta \alpha_{q}\right) \mathrm{d} V_{0} \\
& +\int_{l_{0}} \frac{\partial w_{i}}{\partial \alpha_{p}} \frac{\partial w_{j}}{\partial \alpha_{q}} \delta \alpha_{p} \mathrm{~d} \alpha_{q} C_{i j}^{I} \mathrm{~d} l_{0}+\int_{l_{0}} t_{i} \frac{\partial^{2} w_{i}}{\partial \alpha_{p} \partial \alpha_{q}} \delta \alpha_{p} \mathrm{~d} \alpha_{q} \mathrm{~d} l_{0} \text { in } \Omega
\end{aligned}
$$

The introduction of $\widetilde{\boldsymbol{L}}$ in Eq. (16) modifies the equilibrium equations according to the relation $\delta \widetilde{\Pi}\left(\delta u_{k i}, \delta \alpha_{p}, \delta \beta_{r i}\right)=\delta \Pi\left(\delta u_{k i}, \delta \alpha_{p}\right)+\delta \Pi^{*}\left(\delta \beta_{r i}\right)$, with the term $\delta \Pi^{*}\left(\delta \beta_{r i}\right)$ being calculated as:

$$
\delta \Pi^{*}\left(\delta \beta_{r i}\right)=\int_{V_{0}} \tau_{i j} \frac{\partial B}{\partial \xi_{r}} \overline{\left(\frac{\partial \xi_{r}}{\partial X_{l}}\right)} \frac{\partial X_{l}}{\partial x_{j}} \frac{\operatorname{det}\left[\frac{\overline{\partial X}}{\partial \xi}\right]}{\operatorname{det}\left[\frac{\partial X}{\partial \xi}\right]} \delta \beta_{r i} \mathrm{~d} V_{0}
$$

and, therefore, a corresponding term $\mathrm{d} \delta \Pi^{*}(\mathrm{~d} \boldsymbol{\beta}, \delta \boldsymbol{\beta})$ follows directly.

\subsection{Stress continuity condition}

A strong form of the continuity condition is here proposed and tested (for an averaged form, without modification of the constitutive law, see the work on SKON carried out by Oliver's group [12,14,18,33,34]).

This is accomplished by modifying the constitutive stress $\tau^{C}$ by the introduction of a symmetric tensor $\boldsymbol{A}$ as follows ( $\tau$ represents the equilibrium satisfying stress, in the sense of Eq. 20):

$\tau=\tau^{C}+h \boldsymbol{A}$

$\overline{\boldsymbol{t}}=h \tau^{C} \boldsymbol{F}^{-T} \boldsymbol{N}+h \boldsymbol{A} \boldsymbol{F}^{-T} \boldsymbol{N}$

$\boldsymbol{S}^{T} \boldsymbol{F}^{-1} \boldsymbol{A} \boldsymbol{F}^{-T} \boldsymbol{S}=0$

The equation (29b) represents the stress continuity condition for all integration points belonging to the element's domain, which are close enough to the crack so the condition applies. This condition is also implied, in an averaged form, by SKON formulation.

Equation (29c) represents the absence of contribution of the crack for the normal stress component in the direction of the crack path.

The constitutive Kirchhoff stress, $\tau^{C}$ is obtained here by linear elastic law such as $\tau^{C}=\boldsymbol{C}^{\tau}: \boldsymbol{\varepsilon}$ where $\boldsymbol{\varepsilon}$ is the Almansi strain tensor, in a consistent notation with the previous rate equation (24) written for the Truesdell rate.

The notation $\overline{\boldsymbol{t}}$ in (29b) refers to the averaged stress vector at the crack.

The solution of the system of equations represented by Eqs. (29b, 29c) for the scalar components of $\boldsymbol{A}$ is carried out according to Eq. (30), where use is made of the notations $\boldsymbol{s}=\boldsymbol{F}^{-T} \boldsymbol{S}$ and $\boldsymbol{n}=\boldsymbol{F}^{-T} \boldsymbol{N}$.

$$
\left[\begin{array}{ccc}
n_{1} & n_{2} & 0 \\
0 & n_{1} & n_{2} \\
s_{1}^{2} & 2 s_{1} s_{2} & s_{2}^{2}
\end{array}\right]_{i k-1}\left\{\begin{array}{l}
A_{11} \\
A_{12} \\
A_{22}
\end{array}\right\}_{i k}=\left\{\begin{array}{c}
\overline{\boldsymbol{t}}-\tau^{C} \boldsymbol{n} \\
0
\end{array}\right\}_{i k-1}
$$

As a measure to enforcing symmetry (which is lost with SKON), both the second member and the coefficient matrix in (30) are evaluated in the previously converged step $(i k-1)$.

\section{Particular constitutive relations for the embedded crack}

\subsection{Fracture criterion: initiation}

If the embedded crack has not initiated in a given element, which occurs for $h=0$, the Rankine criterion for crack initiation is tested:

$\check{\tau}_{1} \geq \tau_{1 c}$ 
where $\tau_{1 c}$ is the maximum allowable positive Kirchhoff principal stress (the Kirchhoff tensile strength) and $\check{\tau}_{1}$ is the following quantity:

$\check{\tau}_{1}=\frac{1}{V_{0}} \int_{V_{0}} \max \left(0, \tau_{1}\right) \mathrm{d} V_{0}$

with $\tau_{1}$ being the maximum principal Kirchhoff stress field.

Variations of this type of fracture criterion are widely employed in the context of discontinuity representations $[7,10,11,13,17,27]$.

A definition of $h$ can be exposed using the maximum value of $\check{\tau}_{1}$ during the deformation history:

$h=H\left[\max _{\text {history }}\left(\check{\tau}_{1}\right)-\tau_{1 c}\right]$

The function $H$ in definition (33) is the unit step function:

$H[x]= \begin{cases}0 & x<0 \\ 1 & x>0\end{cases}$

The calculation of the natural coordinates $\xi_{Q}$ of an arbitrary point $Q$ located on the crack initiation point is carried out using a relation where $\xi_{X}$ represents the natural coordinates of a point $X \in \Omega_{1} \cup \Omega_{2}$ :

$\xi_{Q}=\frac{\int_{V_{0}} \max \left(0, \tau_{1}\right) \xi_{X} \mathrm{~d} V_{0}}{V_{0} \check{\tau}_{1}}$

In a distinct form, the calculation of the material coordinates of the normal vector, $\boldsymbol{N}$, is accomplished according to $(j$ counts the integration points in each element and $i$ counts the elements in the neighborhood of the initiated element):

$\boldsymbol{N}=\frac{\sum_{i=1}^{n e l} \sum_{j=1}^{4} N_{1} W(r)}{\sum_{i=1}^{n e l} \sum_{j=1}^{4} W(r)}$

where $W(r)$ represents an exponential weight function centered in $Q, r$ represents the distance between the point $Q$ and the corresponding neighborhood point. Finally, $N_{1}$ represents the second Piola-Kirchhoff stress principal direction field.

An weighted average is used in (36) to attenuate the fact that although we aim at a $C^{0}$ continuous crack path, the stress field is discontinuous between element's edges. Similar arguments were employed in reference [16] in the context of the hyperbolicity indicator calculation.

One feature in (36) is that the radius of support of function $W$ is taken as $R=2 \times$ the element characteristic length, see Fig. 2, also indicated in ref [16] for a leastsquare average technique.

The material coordinates of $\boldsymbol{S}$ are evaluated by the vector product $\boldsymbol{S}=\boldsymbol{N} \times \boldsymbol{e}_{3}$ where $\boldsymbol{e}_{3}=\{0,0,1\}^{T}$.

With the quantities $\xi_{Q}$ and $N$ defined by relations (35) and (36) respectively, the material coordinates of points $O$ and $P$ can be calculated by the intersection of a straight line defined by the point $Q$ and the vector $\boldsymbol{S}$ with each element's outer boundary $\partial \Omega_{1} \cup \partial \Omega_{2}$. This task is carried using material coordinates.
As a result of this calculation, the natural coordinates of points $O$ and $P$ can be identified as $\xi_{O}$ and $\xi_{P}$ respectively.

Finally, the spatial coordinates $\boldsymbol{n}$ and $\boldsymbol{s}$ (the spatial counterparts of $\boldsymbol{N}$ and $\boldsymbol{S}$ ) can be directly evaluated using the natural coordinates of points $O$ and $P$.

\subsection{Crack path}

After the initiation of a crack, two tails can propagate. Hence, for a two crack problem, four tails exist.

After the formation of each tail, propagation is carried out by using the normal given by Eq. (36) but now centered on each crack tip.

As point $O$ is taken as the previous crack tip on the same tail, $P$ is directly calculated from $N$ (see Fig. 2). In this figure, the radius $R$ is employed to average the stress tensor used in the evaluation of $\boldsymbol{N}$. Its value is taken here as two element sides, but larger values may be needed for other problems, as recently observed in ref [35] in the context of the extended finite element method. It is clear that although crack path is continuous along element edges (see Fig. 2), both the opening displacement magnitude and direction are not.

\subsection{Crack compliance}

If the condition $h=1$ is satisfied (see Sect. 2.4), the stress vector $\boldsymbol{t}$ introduced in Eq. (19) is independent of the bulk constitutive law (see also references [12,36]). This stress vector should be related to the crack state (i.e. $\boldsymbol{w}$ ).

To represent $\boldsymbol{t}$ as a function of $\boldsymbol{w}$, it is convenient to introduce the local spatial coordinates of $\boldsymbol{t}$ in the frame $\{\boldsymbol{s}, \boldsymbol{n}\}$ :

$t_{1}=\boldsymbol{t} \cdot \boldsymbol{s}$

$t_{2}=\boldsymbol{t} \cdot \boldsymbol{n}$

With the help of a kinematic variable, $\kappa$, the following local constitutive law is assumed for the coordinates $t_{1}$ and $t_{2}$ :

$$
\left\{\begin{array}{l}
t_{1} \\
t_{2}
\end{array}\right\}=\left\{\begin{array}{c}
A_{1}(\kappa) w_{1}\left(\alpha_{1}, \alpha_{2}, \alpha_{3}\right) \\
{\left[A_{2}(\kappa) H\left(w_{2}\right)+\rho H\left(-w_{2}\right)\right] w_{2}\left(\alpha_{1}, \alpha_{2}, \alpha_{3}\right)}
\end{array}\right\}
$$

where $A_{i}(\kappa)$ with $i=1,2$ represent given functions of the kinematic variable $\kappa$ :

$A_{1}(\kappa)=d_{\text {int }} \exp \left[\ln \left(\frac{d_{1}}{d_{\text {int }}}\right) \kappa\right]$

$A_{2}(\kappa)=\frac{\tau_{1 c}}{\kappa} \exp \left(-\frac{\tau_{1 c}}{G_{f}} \kappa\right)$

These particular forms (39a) and (39b) are a consequence of the adoption of an anisotropic damage evolution law $[10,27]$. Conclusions regarding the accuracy in 
Fig. 2 Crack path algorithm: forced path continuity and averaged normal vectors at the crack tip

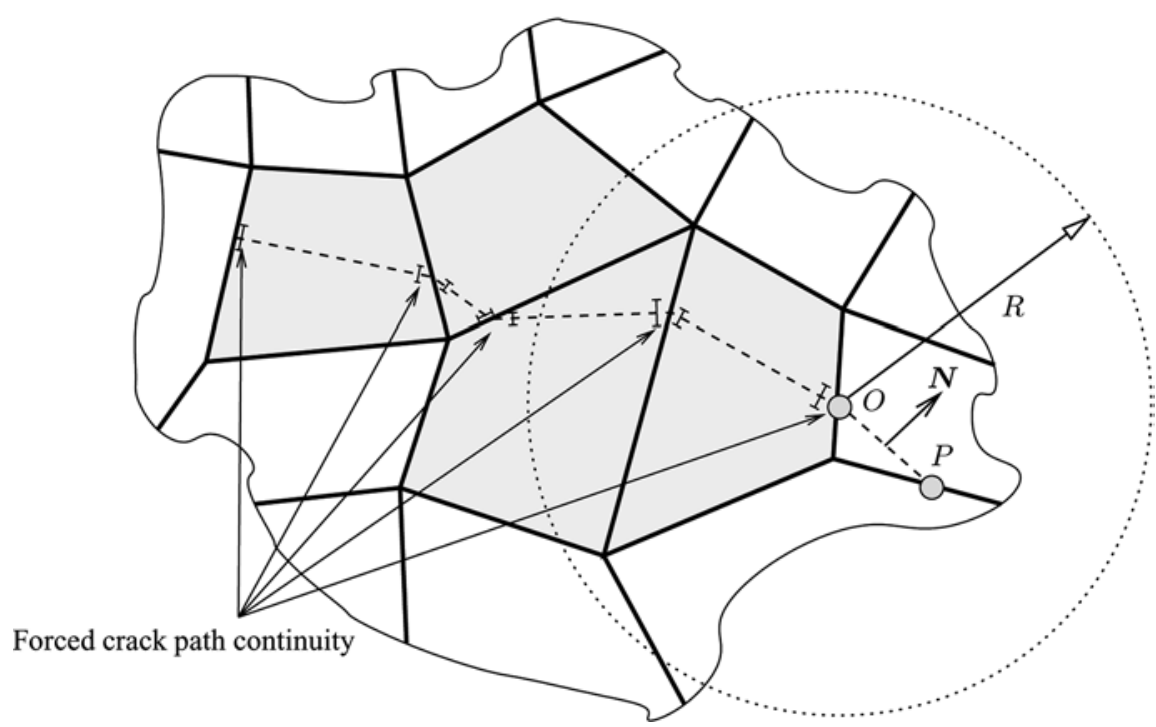

mixed mode brittle fracture appear favorable to this law (see reference [10] for mixed mode tests).

In addition, reference [27] shows that for the singleedge notched beam test a constant shear stiffness is unsuitable. Constant shear stiffness was employed, among others, in reference [7].

In Equation (38) $\rho$ is a penalty parameter, which is included to ensure the approximate satisfaction of inequalities (18). The value of $\rho$ is taken here as the absolute value of the interface compliance before initiation:

$\rho=\frac{\tau_{1 c}^{2}}{G_{f}}=-\lim _{w_{2} \mapsto 0^{+}} \frac{\partial t_{2}}{\partial w_{2}}$

In Equation (39b), the term $G_{f}$ represents the fracture energy.

The kinematic variable $\kappa$ is calculated using the historical evolution of the normal displacement $w_{2}$ :

$\kappa=\max _{\text {history }}\left[\max \left(0, w_{2}\right)\right]$

Regarding the shear stiffness, the property $d_{\text {int }}$ in Eq. (39a) represents the initial shear stiffness of the crack and $d_{1}$ represents the shear stiffness of the crack for $\kappa=1$. The condition $d_{\text {int }}>d_{1}$ should be satisfied.

Some aspects of the particular constitutive law (38) are:

- The stress coordinates $t_{1}(\kappa)$ and $t_{2}(\kappa)$ contain unique global maximum values in the interval $\kappa \in[0,+\infty$ [ for $\kappa=0$. The maximum values are $t_{1_{\max }}=d_{\text {int }}$ and $t_{2_{\max }}=\tau_{1 c}$

- It is possible to identify the fracture energy $G_{f}$ with the energy release rate $G_{I}$ ( $I$ indicates the opening mode). Accordingly, for proportional loading, $G_{I}=G_{f}=\int_{0}^{+\infty} t_{2} \mathrm{~d} \kappa$

- The exponential form for this law is not unique. Other authors (e.g. [37]) have used linear softening behavior, for example. However, the exponential form circumvents the use of another branch in the constitutive law, which constitutes an advantage for NewtonRaphson algorithms. A number of specialized softening functions for concrete, polymethyl-methacrylate (PMMA) and pearlitic steel was presented in reference [36]

Despite the conclusion that, for small cracked areas, the shape of the softening function is of relatively minor importance [36], more general situations require experimental determination of the functions, using inverse methods (see reference [36] for such procedures).

The determination of the term $C_{i j}^{l}$ introduced in Eq. (23) follows:

$C_{11}^{l}=A_{1}$

$C_{12}^{l}=A_{1} w_{1} \ln \left(\frac{d_{1}}{d_{\text {int }}}\right) \frac{\partial \kappa}{\partial w_{2}}$

$C_{21}^{l}=0$

$$
C_{22}^{l}=\left[1-w_{2}\left(\frac{\tau_{1 c} \kappa+G_{f}}{G_{f} \kappa}\right) \frac{\partial \kappa}{\partial w_{2}}\right] A_{2} H\left(w_{2}\right)+\rho H\left(-w_{2}\right)
$$

Another feature in the present formulation for softening is whether the tangential displacement at the crack, $w_{1}$, should contribute to the internal variable $\kappa$.

General considerations regarding both the anisotropic damage law and the compression softening behavior have been carried out in reference [38].

An alternative to the consideration of a distinct law for $\boldsymbol{t}$ involves the introduction of a fracture initiation criterion based on the nullity of the acoustic tensor determinant (reference [39] presents a general technique to accomplish that non-trivial task) and the consideration of an interface constitutive law supported by the 
Fig. 3 Three point bending of a cracked beam (all dimensions are in $\mathrm{m}$ )

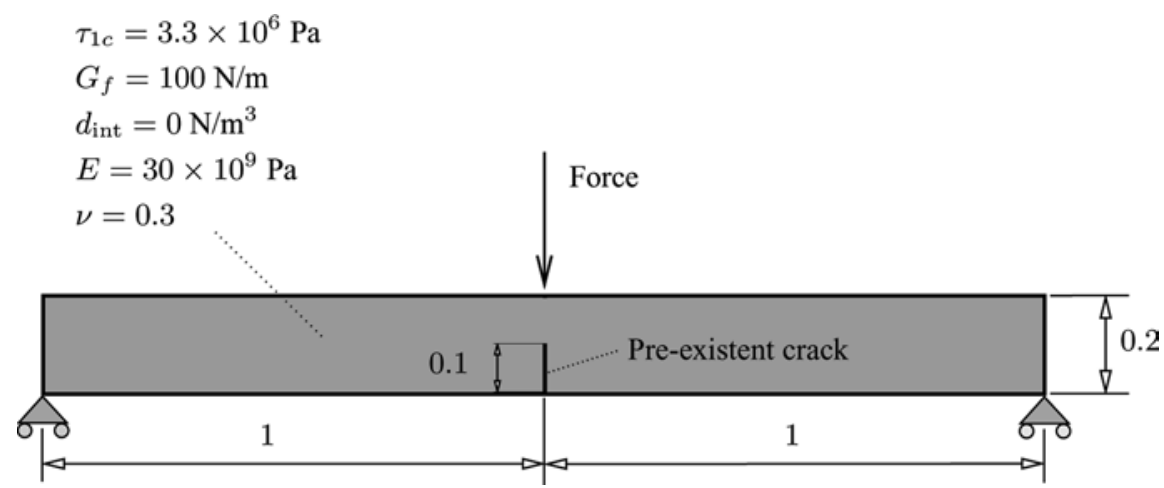

bulk behavior (see references $[2,6,12,16,33,34,40$, 41]).

It is observable that the treatment of ductile fracture in metals can also be carried with an interface constitutive law, but the stress triaxiality parameter should be considered (see reference [42]) in the interface law.

\section{Numerical examples}

The proposed simplified model requires the following properties:

- The tensile strength, $\tau_{1 c}$

- The fracture energy, $G_{f}$

- The initial shear stiffness, $d_{\text {int }}$

- The shear stiffness for $k=1, d_{1}$

- The elasticity modulus, $E$

- The Poisson coefficient, $v$

Fig. 4 The deformed meshes (with a scale factor of 100) corresponding to two distinct mesh densities
Regarding the element quadrature, for the domain $\Omega_{1} \cup \Omega_{2}$ a standard Gaussian quadrature is adopted, a fact that is clearly distinct from the techniques employed in references $[14,18]$ in the context of embedded displacement discontinuities, and in references $[15,16]$, in the context of the extended finite element method.

For the crack domain, $\Gamma$, a nodal integration rule is employed.

The Newton-Raphson method with line-search is adopted in the following analyses. These were carried out in SIMPLAS, a code created by the first author of this work.

\subsection{Three point bending: mode $I$ small strain test}

This test consists in the bending of a cracked beam. A pre-existent crack is located at the mid span and propagates upwards as the load magnitude increases. Fig. 3 shows the geometry, boundary conditions and material properties of the model.

The geometrical data and the boundary conditions are taken from references $[43,11]$.

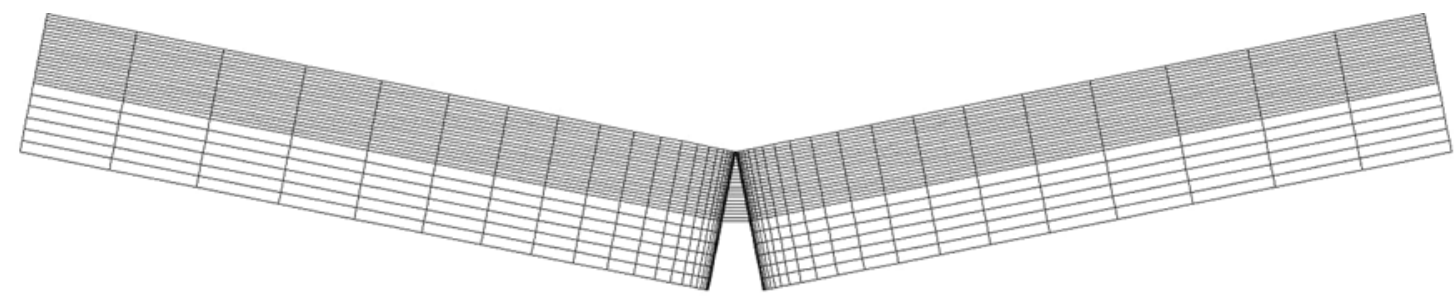

(a) Sparse mesh

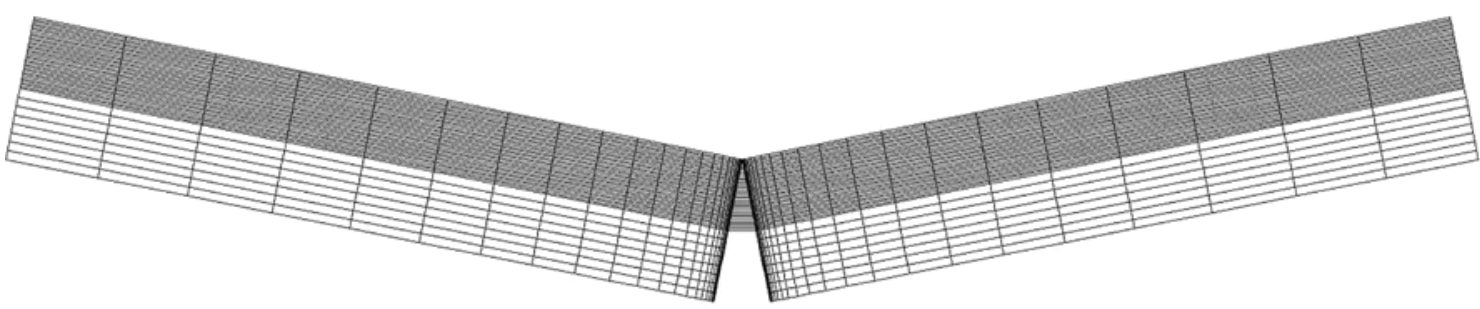

(b) Refined mesh 
Fig. 5 The force-displacement plot for the three point bending test (see Fig 3). The experimental points were reproduced from those exposed in reference [11]

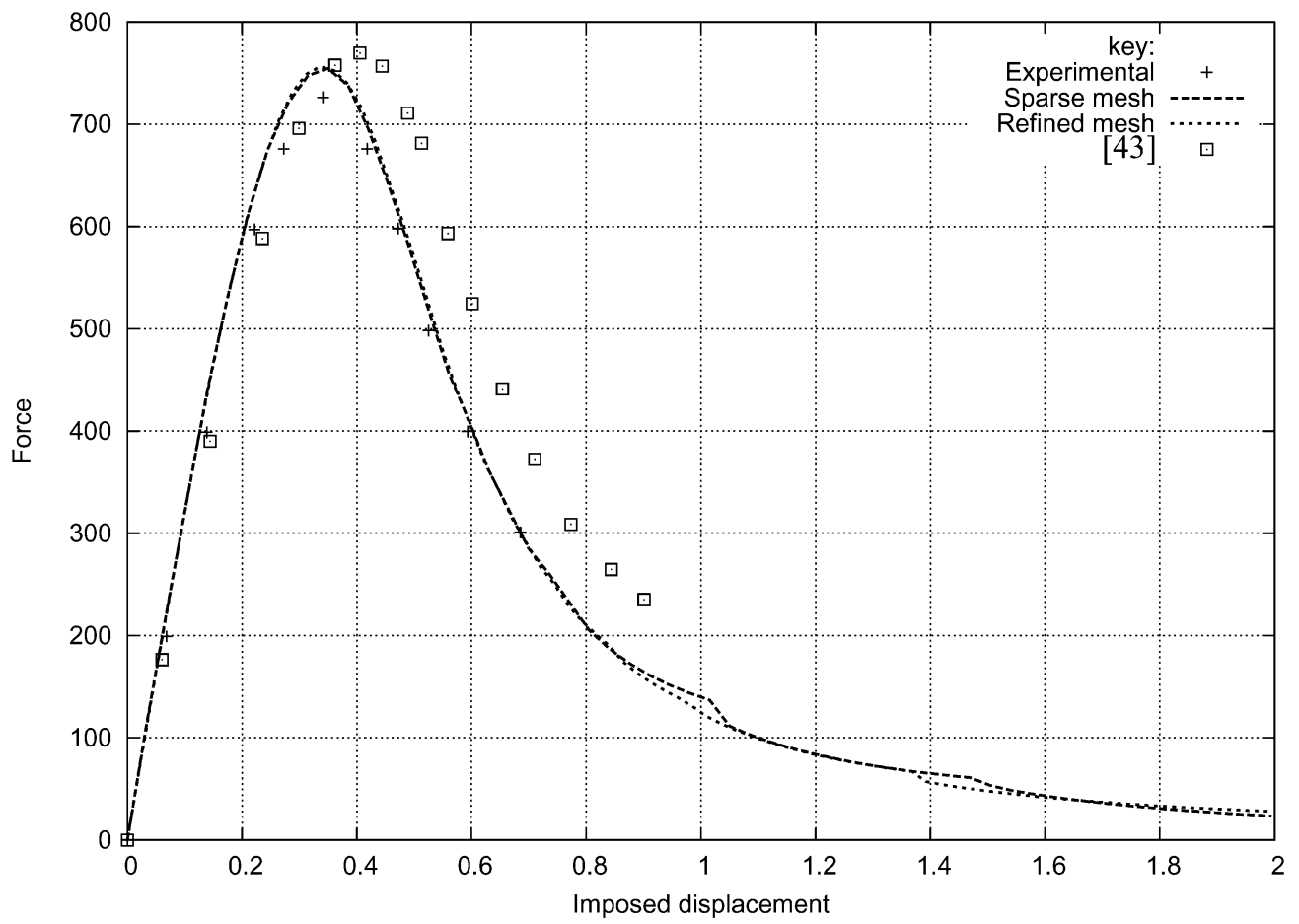

Two mesh densities are used, with the purpose of inspecting some possible mesh size dependency of the crack propagation. The deformed meshes for a $2 \mathrm{~mm}$ mid span displacement (scaled $100 \times$ ) are represented in Fig. 4. The coarse mesh contains 1268 elements and the refined mesh contains 2099 elements.

The force magnitude ( $F$ in Fig. 3 ) is plotted as a function of the mid-span transversal displacement in Fig. 5. Along with the present results, some experimental values obtained by Petersson (see reference [11] for further details) are also presented. There is a very close agreement between the numerical results and the

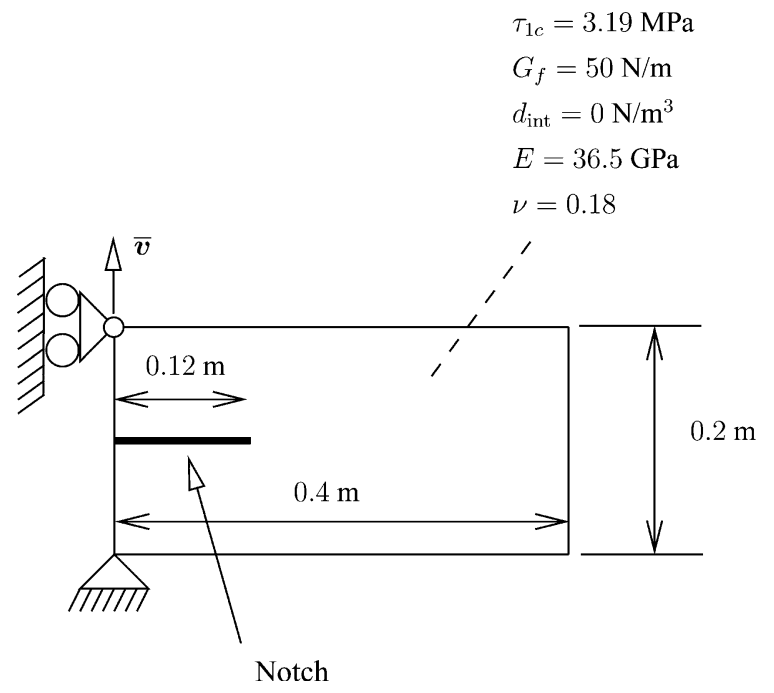

Fig. 6 Double cantilever beam (DCB) with a notch, see also reference [44] experimental ones. For comparison, the use of a gradient regularized media (see reference [43]) is not sufficient to represent the experimental results.

\subsection{DCB test}

This example shows a comparison between the results obtained using other numerical technique (see reference [44]) and the proposed model using embedded discontinuities. It consists of a double cantilever beam loaded in mode $I$. In this problem, the crack path is unstable [44]. Complete details of this problem can be consulted in reference [44].

A schema with the geometry, material properties and boundary conditions is depicted in Fig. 6.

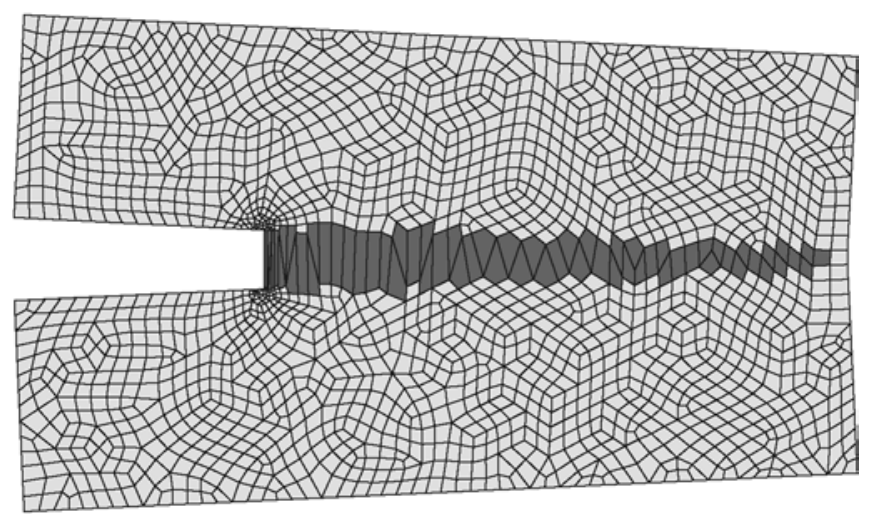

Fig. 7 DCB test: deformed mesh and set of elements containing the crack path, for the unstructured mesh. A displacement magnification of $50 \times$ is employed 
Fig. 8 Load-displacement curve for the DCB test with unstructured mesh. Comparison with reference [44]. The notation $\mathrm{R}$ indicates activation of rotation $\left(\alpha_{3}\right)$ and the notation $\mathrm{S}$ indicates activation of the stress correction, equation $29 \mathrm{a}$

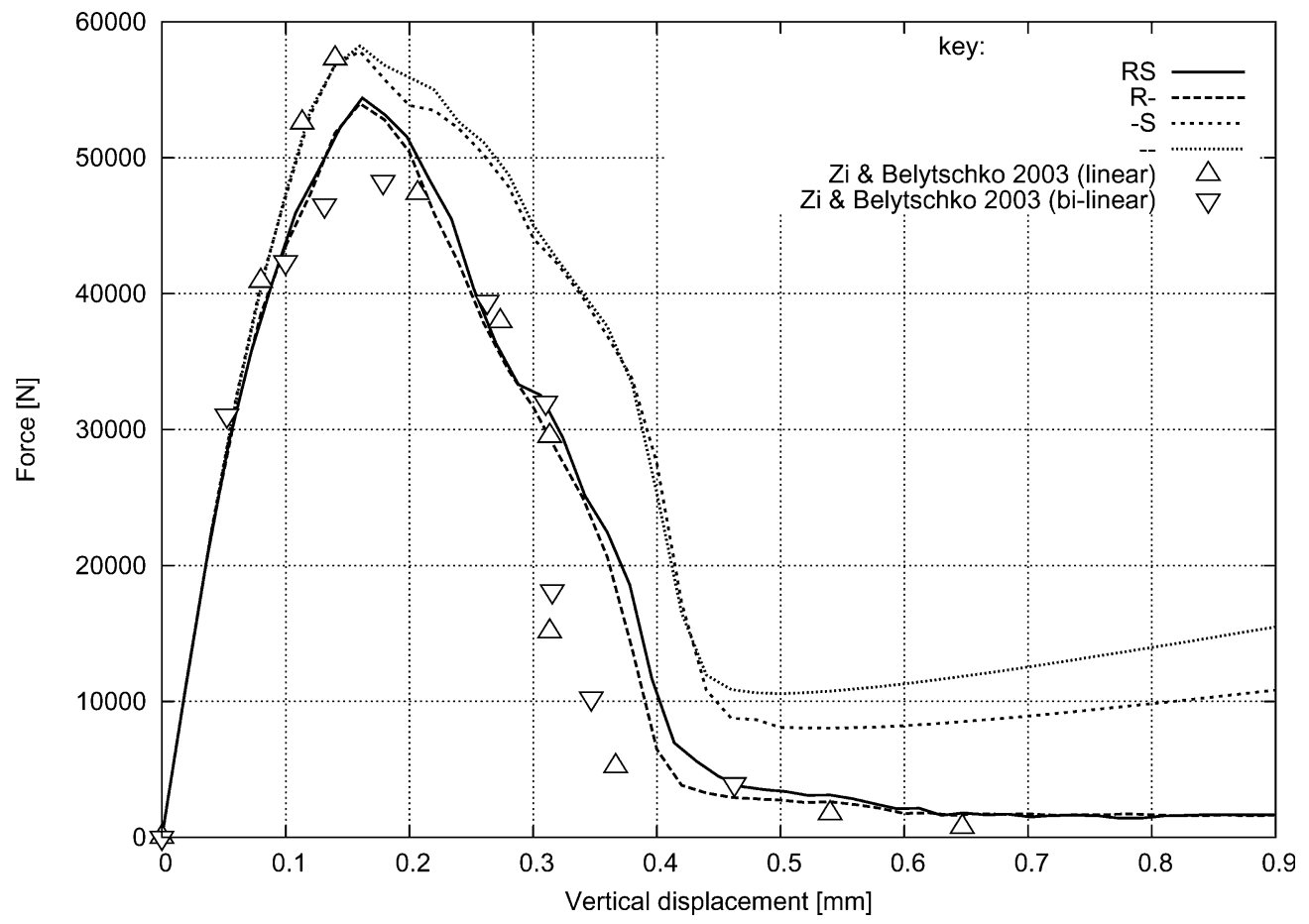

Firstly, a highly unstructured mesh is adopted and the corresponding results are inspected.

The deformed mesh, represented with a magnification of $50 \times$, along with the set of elements containing the crack path is shown in Fig. 7 (1937 elements are present in the mesh). No special refinement is employed in the crack zone nor at the notch tip.

The load-displacement curve for the unstructured mesh is represented in Fig. 8, along with the corresponding results from reference [44] for both linear and bi-linear softening cohesive law. In this figure, locking effects are clear for formulations without rotation of the crack faces (both -S and - in Fig. 8). The effects of stress enhancement and rotation are notorious in the post-stable region.

Despite the mesh quality, it is clear that the results are similar to the ones obtained using other formulation [44].
Fig. 9 Load-displacement curve for the DCB test with structured mesh. Locking is due to mesh distortion induced by deformation. The notation $\mathrm{R}$ indicates activation of rotation $\left(\alpha_{3}\right)$ and the notation $\mathrm{S}$ indicates activation of the stress correction

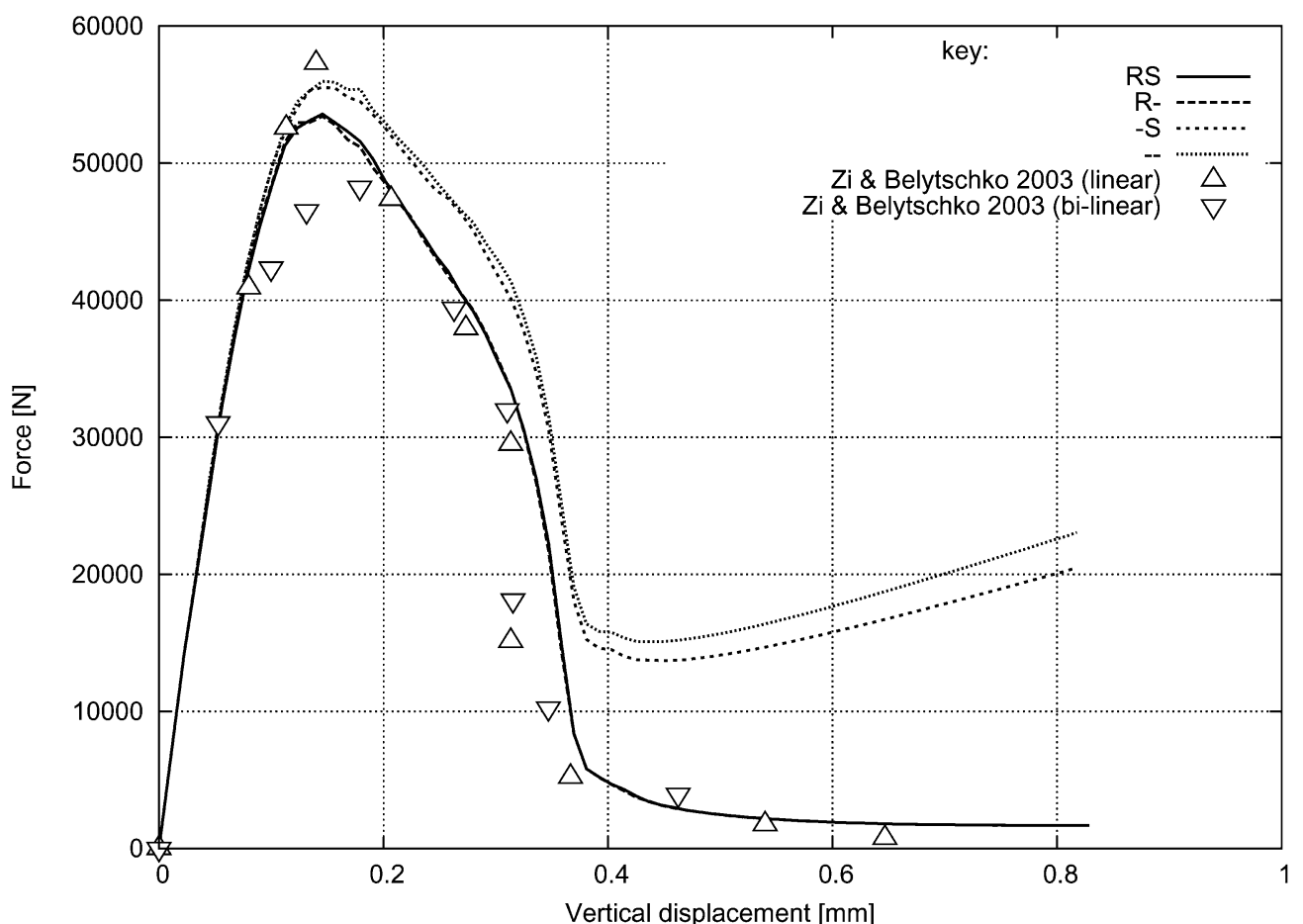




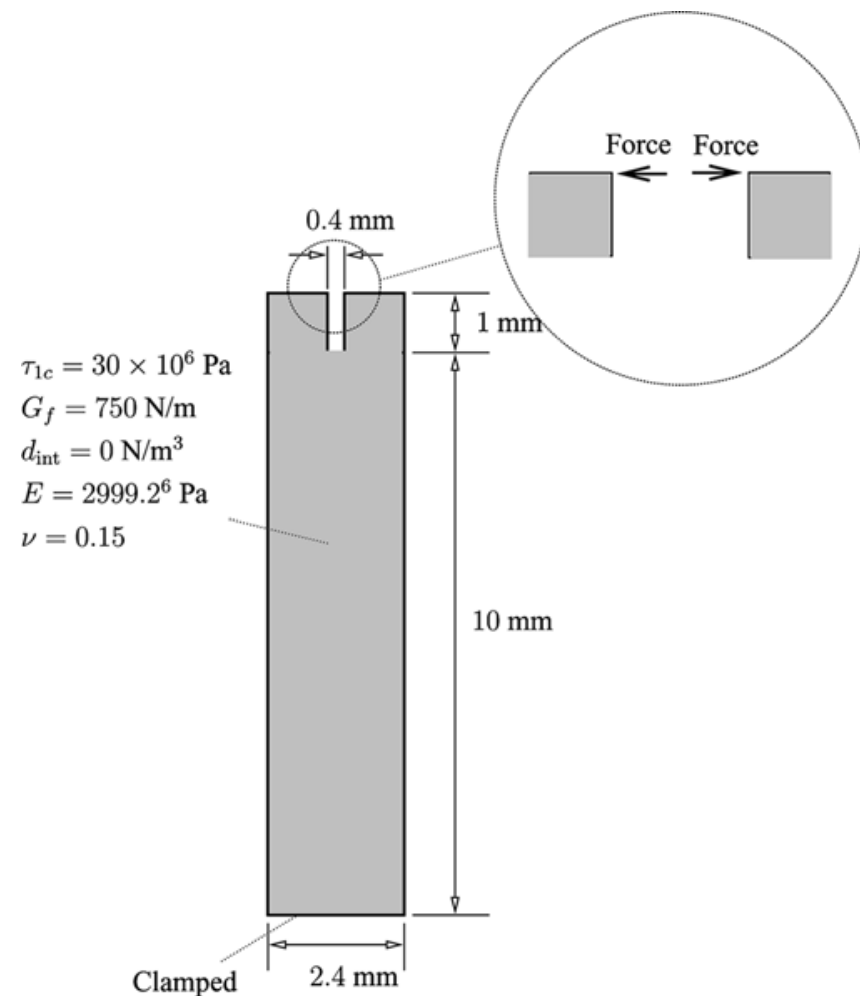

Fig. 10 Geometry, boundary conditions and material properties employed in the debonding analysis

To verify the conclusions by Jirasek regarding the fact that the differences between symmetric and nonsymmetric formulations are only apparent for irregular meshes, a regular mesh is tested. The fact that locking occurs for KOS when the element sides are not aligned
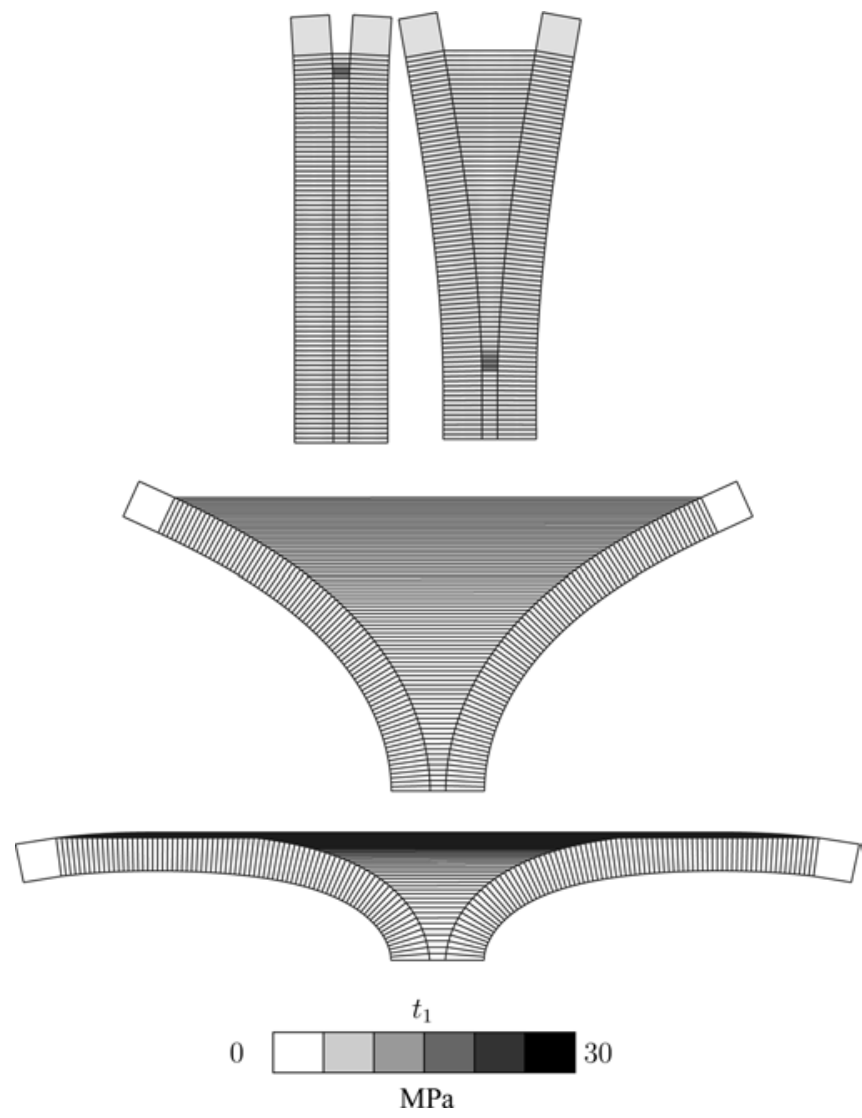

Fig. 11 True deformed meshes corresponding to distinct time steps in the analysis of the debonding problem. The $t_{1}$ stress component contour plot is also presented

was already verified in Fig. 8 for the standard $\operatorname{KOS~}(-)$. However, even in the situation where the initial
Fig. 12 Force-crack opening plot for the debonding problem. Comparison with the results presented in reference [12]

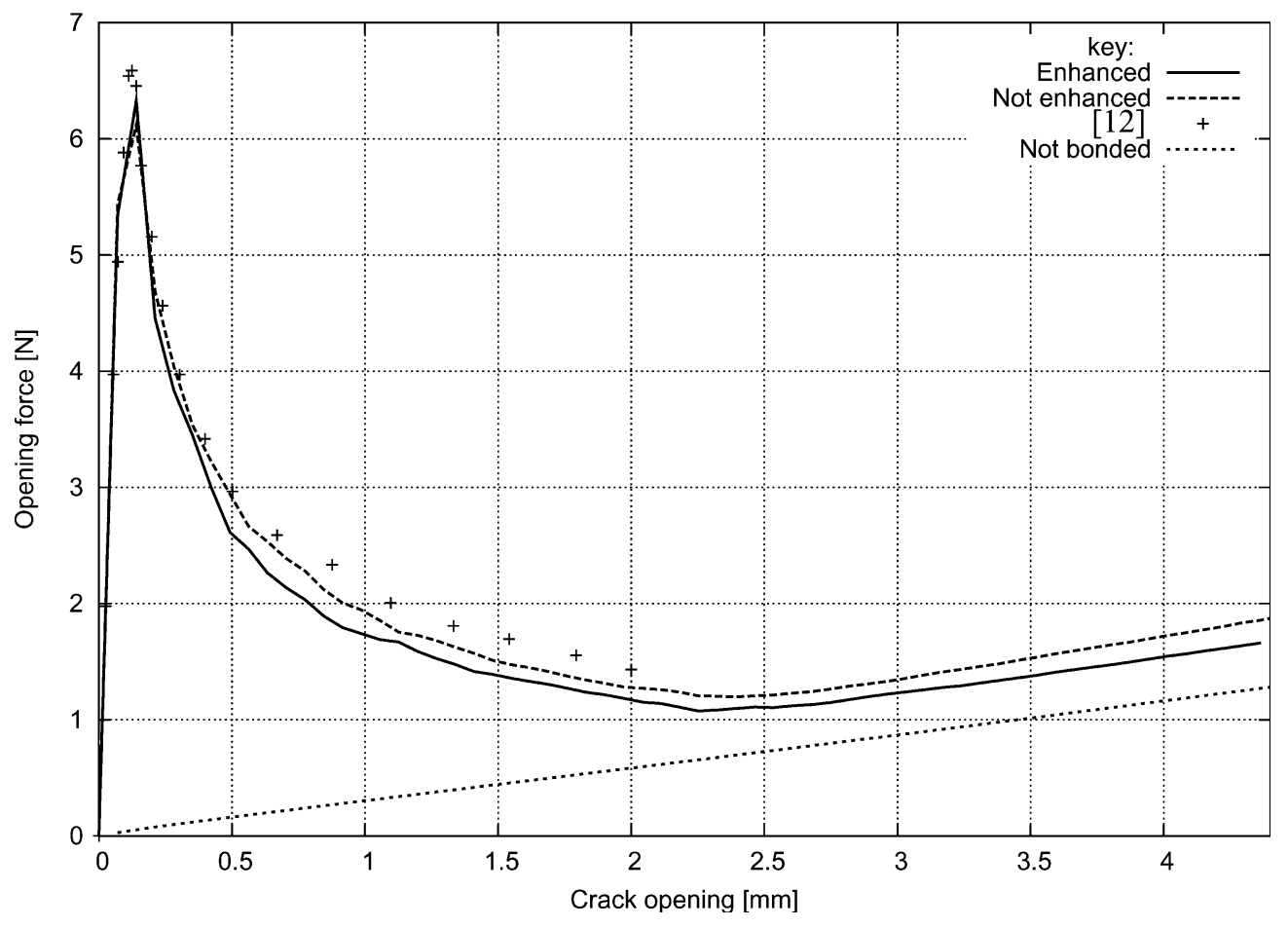




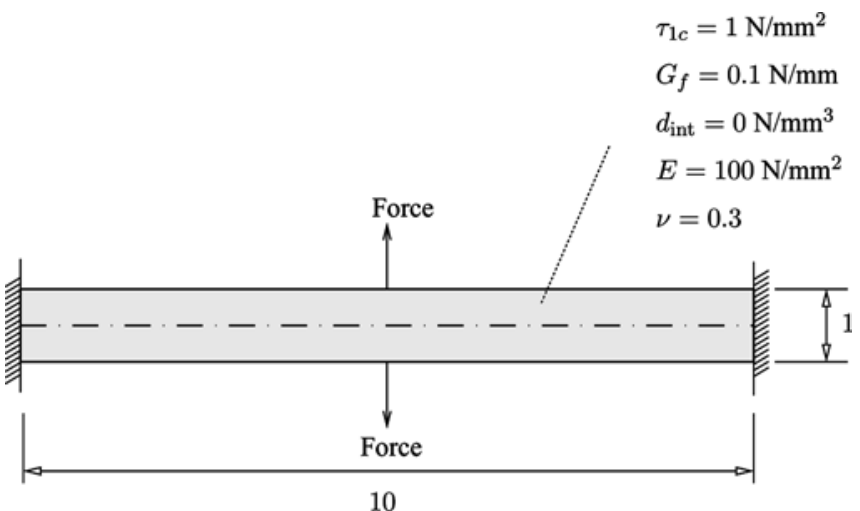

Fig. 13 The clamped beam under central loading (all dimensions are in $\mathrm{mm}$ )

alignment exists, locking occurs due to deformation-induced mesh distortion. This particular aspect, at least in the authors' knowledge, was not verified elsewhere. Fig. 9 illustrates this fact. The locking verified in this figure is even more clear than in Fig. 8 because a coarser mesh is employed. As expected, the results are smoother with this mesh.
Table 1 Single-edge notched beam in mixed mode bending: material properties (see also ref. [34] for other relevant data)

\begin{tabular}{ll}
\hline Property & Value \\
\hline$\tau_{1 c}$ & $3 \times 10^{6} \mathrm{~Pa}$ \\
$G_{f}$ & $0.069 \mathrm{~N} / \mathrm{mm}$ \\
$d_{\text {int }}$ & $2 \times 10^{2} \mathrm{~N} / \mathrm{mm}^{3}$ \\
$d_{1}$ & $1 \times 10^{1} \mathrm{~N} / \mathrm{mm}^{3}$ \\
$E$ & $3.8 \times 10^{10} \mathrm{~Pa}$ \\
$v$ & 018 \\
\hline
\end{tabular}

\subsection{Debonding problem: a finite strain test}

This test is a finite strain analysis of a debonding problem. The geometry, boundary conditions and material properties are presented in Fig. 10.

The analysis purpose is to separate two initially bonded plates by pulling the top notch as depicted in Fig. 10. This test was carried out by Oliver et al. with triangular elements (see reference [12]) and a much more refined mesh. In this example, an evaluation of the enhanced strain term is carried out.

Four distinct steps of the debonding analysis are represented in Fig. 11, where the contour plot of the
Fig. 14 The clamped beam under central loading: deformed mesh $(7 \times 200$ elements). It is important to note that the deformed mesh is not magnified

Fig. 15 Force $[\mathrm{N}]$ versus opening displacement [mm] curves for the clamped beam problem. Results from reference [17] are also represented
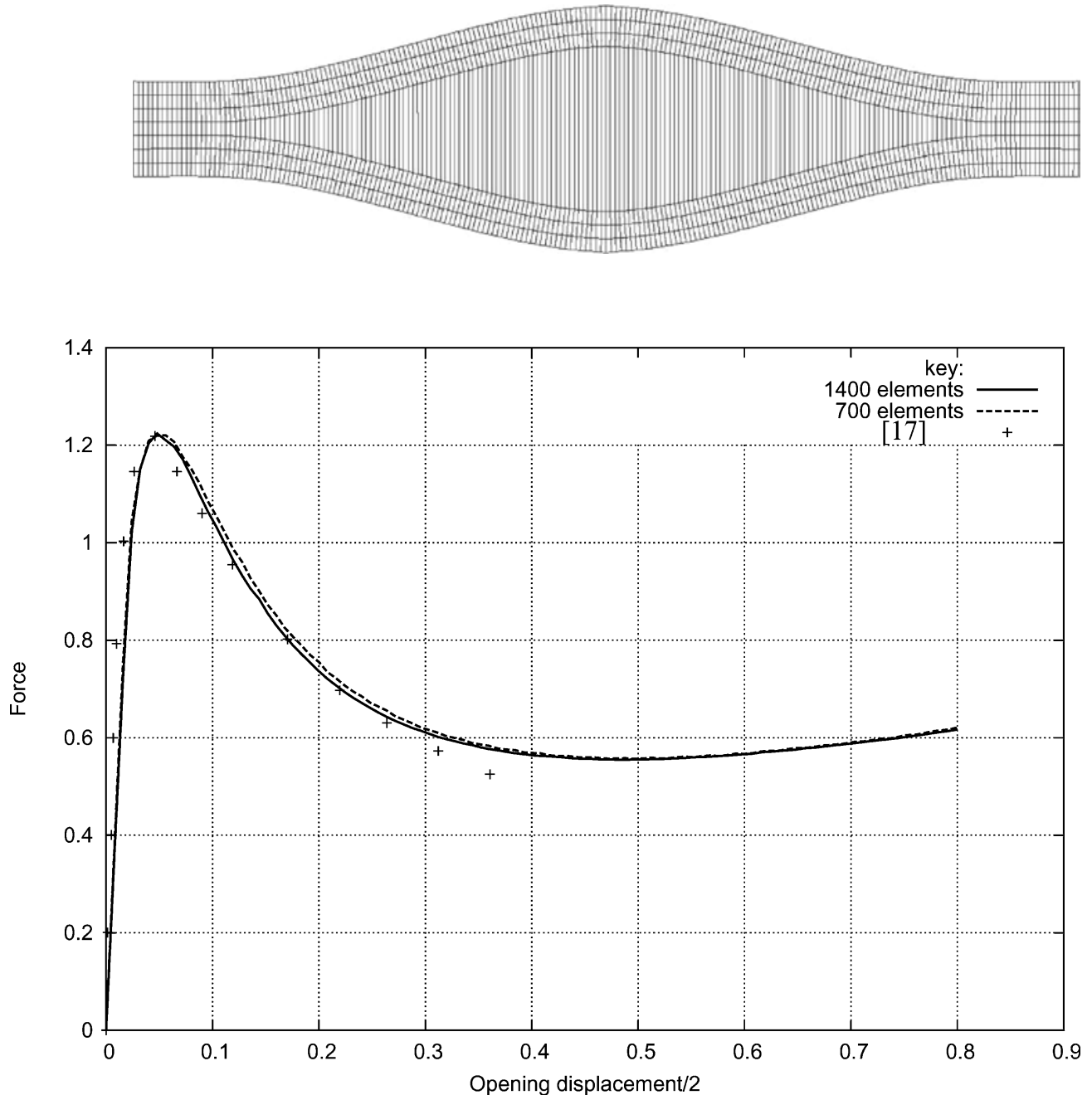
Fig. 16 Initial mesh and two deformed meshes corresponding to the mixed mode bending, with a scale factor of $100 \times$
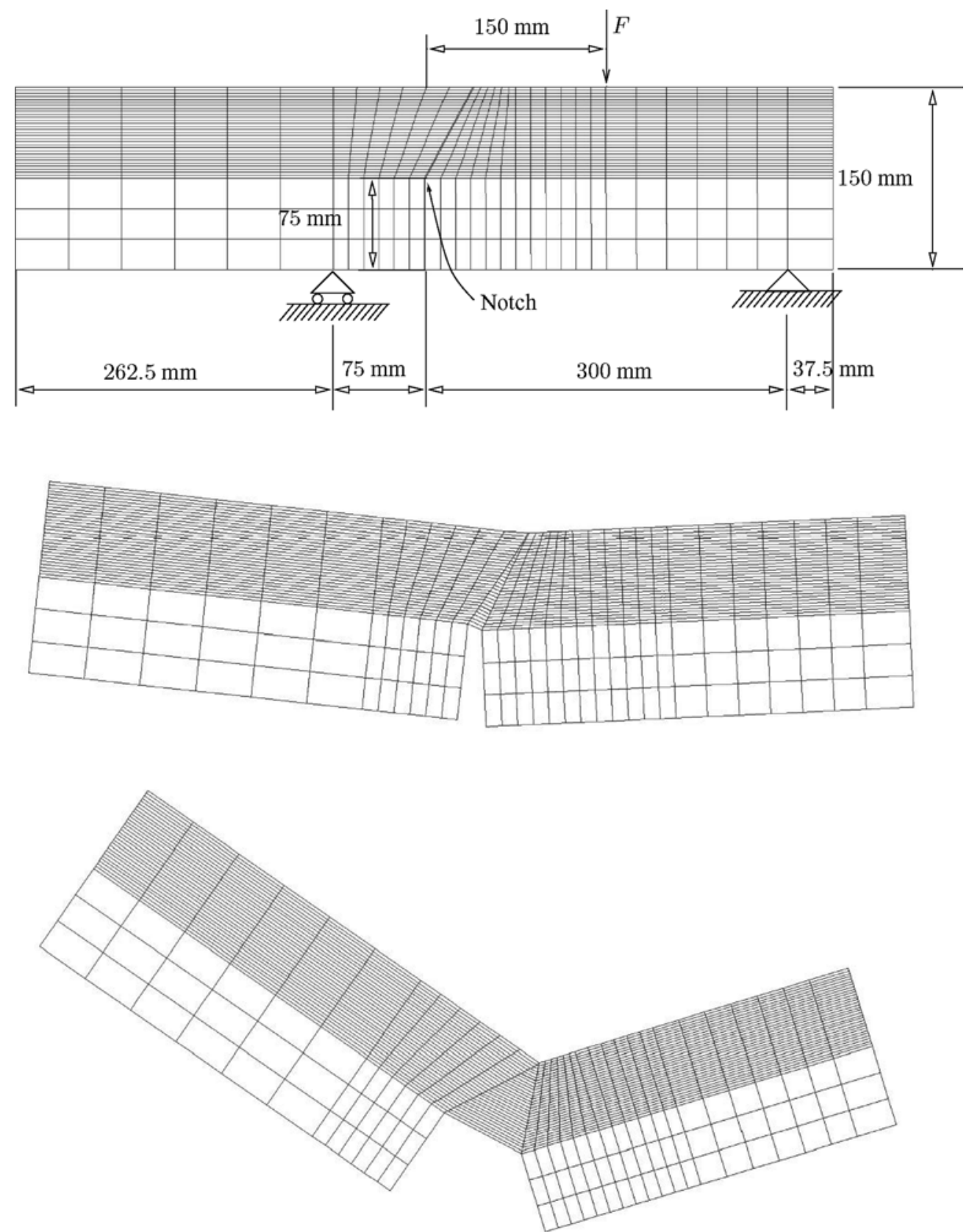

horizontal Kirchhoff stress $\tau_{11}$ is also represented. It is noticeable that the deformed meshes in 11 are not magnified, showing the excellent performance of the proposed algorithms.

Finally, the force-crack opening curve is presented in Fig. 12 and is compared with the numerical results of reference [12]. The values obtained turning off the strain enhancement are also shown for comparison.

An interesting aspect should be noted: the increasing load after most debonding has taken place is due to the clamping of the two arms, as the reaction curve for the non-bonded case shows in Fig. 12.

The effect of the enhanced strain is also clear, with a softer and less steep curve obtained with the enhanced strain term.
5.4 Clamped beam under central loading: mesh size effect

This recent test [17] consists on the analysis of the lateral loading of a clamped-clamped beam without initial notches or cracks.

The geometry, boundary conditions and material properties for this test are represented in Fig. 13 in agreement with the values proposed in reference [17].

For comparison purposes, two mesh densities are tested: $7 \times 100$ and $7 \times 200$ elements. In reference [17] a mesh containing $7 \times 199$ elements was employed.

In both meshes, a central crack initiates for a force of magnitude $F=0.657 \mathrm{~N}$, whereas in reference [17] the crack initiation force was $F=0.45 \mathrm{~N}$. 
Fig. 17 Mixed mode bending: force magnitude as a function of CMOD

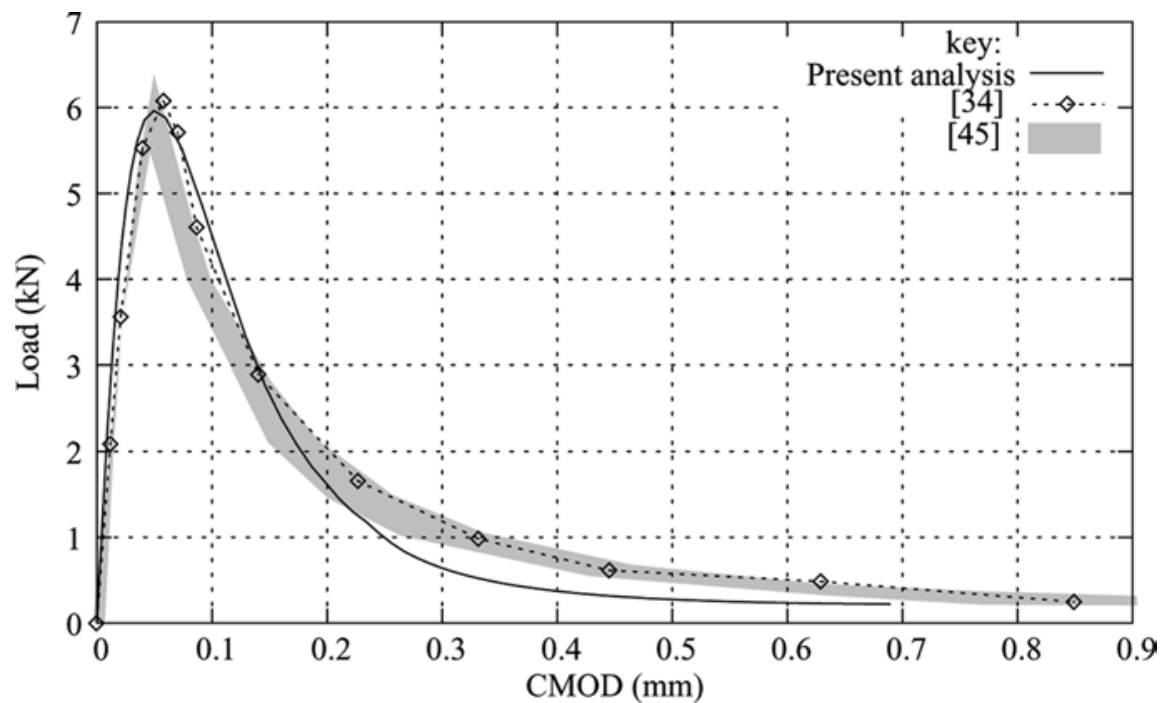

In the present analysis, the total imposed displacement under force was $0.8 \mathrm{~mm}$ whereas in the above reference a total of $0.36 \mathrm{~mm}$ was adopted. This fact shows the high level of robustness which is obtained with the present formulation.

The deformed mesh (for the $7 \times 200$ elements case) is represented in Fig. 14.

For comparison purposes, the force versus opening displacement curves for the two mesh densities are compared with the results obtained in reference [17]

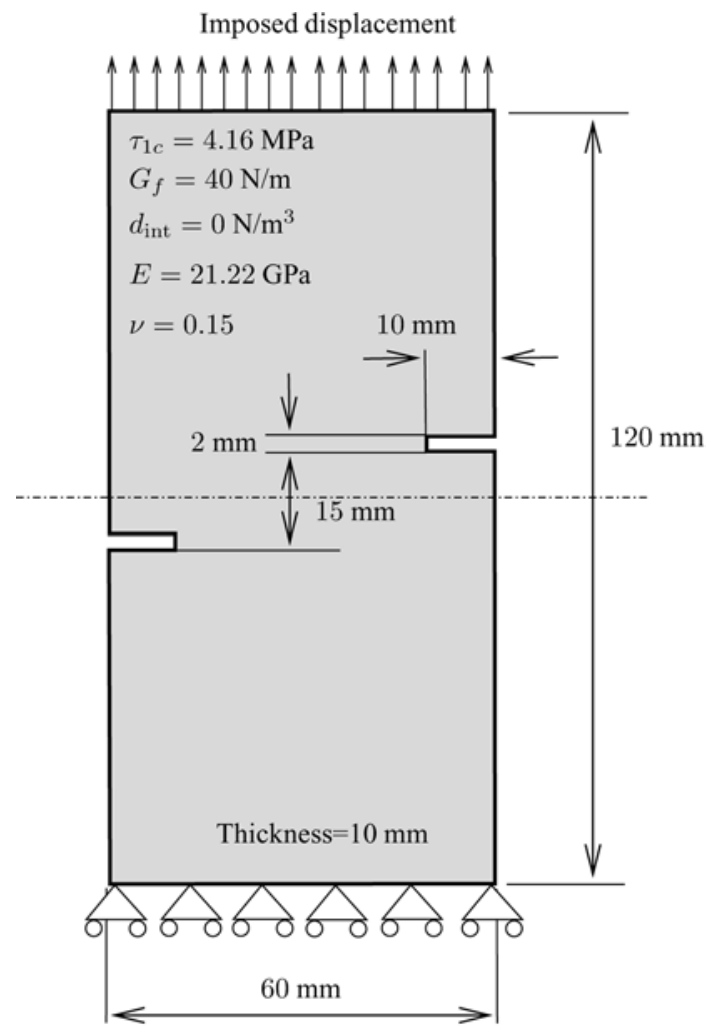

Fig. 18 Relevant data for the double edge-notched tension test where a 1393 element mesh was adopted. Fig. 15 presents the results.

It is clear, from the observation of Fig. 15, that close agreement with the results obtained in reference [17] is

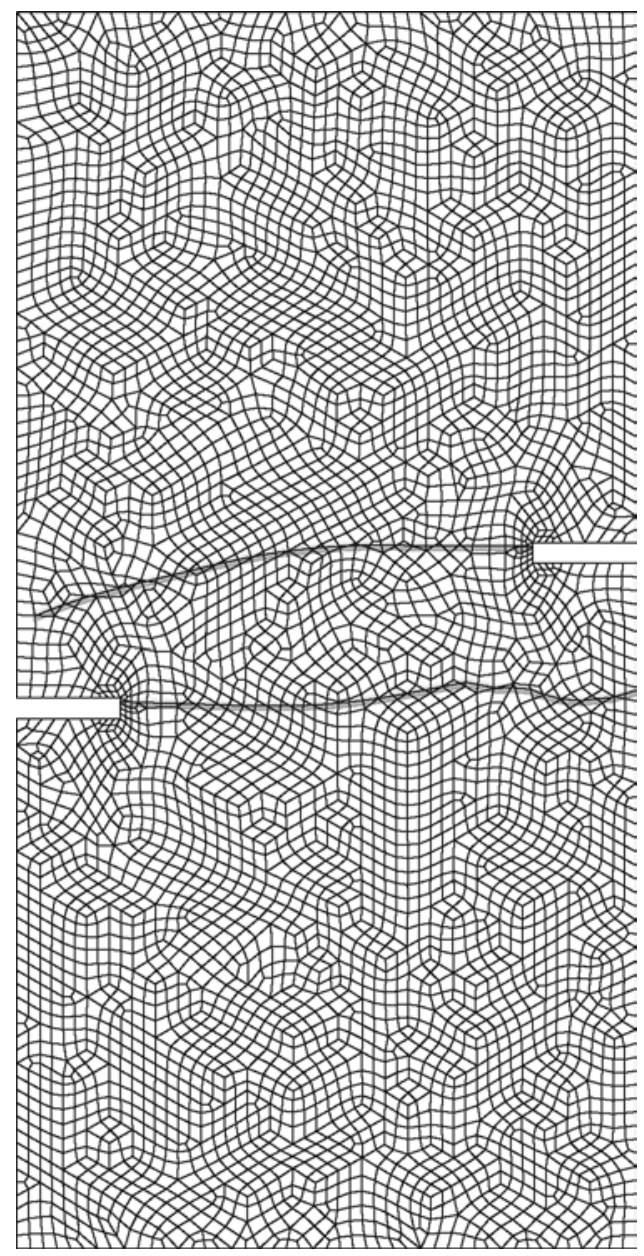

Fig. 19 Crack paths in the double edge-notched tension test 
Fig. 20 Magnified $(60 \times)$ meshes corresponding to $\rho=0$ and $\rho=\tau_{1 c}^{2} / G_{f}$, respectively

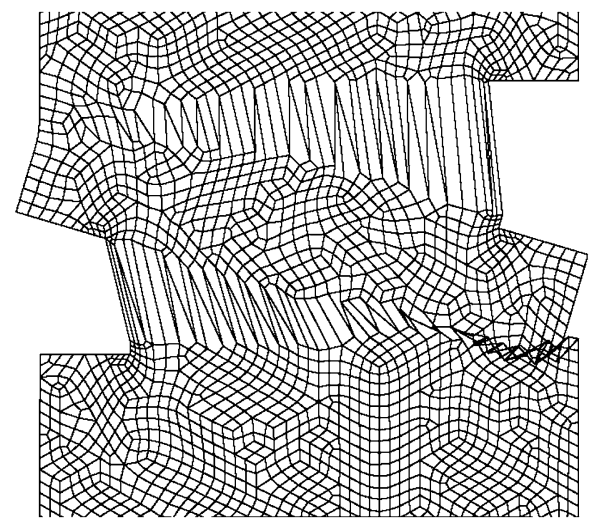

(a) $\rho=0$

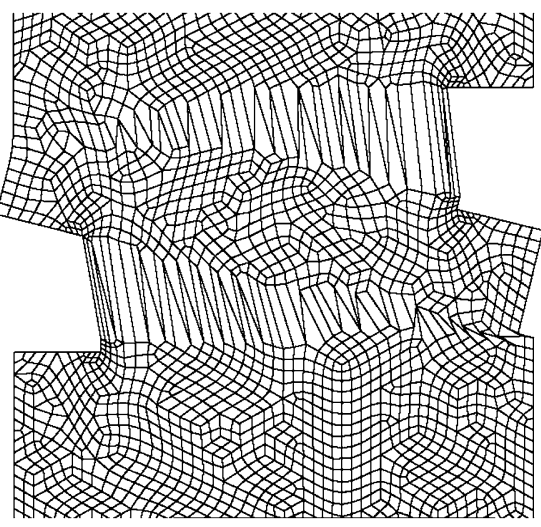

(b) $\rho=\tau_{1 c}^{2} / G_{f}$ verified. Additionally, much higher values of displacement are possible.

One important conclusion can be extracted from this example: with a much coarser mesh (only $7 \times 100$ elements) the results are nearly identical to those obtained in the above reference with almost twice the number of elements.

\subsection{Single-edge notched beam: mixed mode bending}

This benchmark for mixed mode crack propagation consists on the three point bending of a notched beam (an experimental study was carried out in reference [45]). The crack initiates in one of the corners of the notch and propagates upwards diagonally. The benchmark data is presented in references [34, 45].
The present purpose of this test is to verify the robustness of the algorithm in mixed mode fracture using a very coarse mesh (when compared with the one employed in reference [34]) of 1053 elements.

The geometry and boundary conditions can be consulted in reference [34]. The material properties are summarized in Table 1. It is noticeable that the shear stiffness values are not given in the above reference.

A number of deformed (scaled $100 \times$ ) meshes corresponding to distinct time steps is represented in Fig. 16.

The results, presenting the force as a function of the crack mouth opening displacement (CMOD), are given in Fig. 17.

Despite the difference between the two numerical results in the softening zone, the present results are not too far from the experimental values depicted in reference [34] as obtained by Galvez et al
Fig. 21 Load-displacement curve for the double edgenotched tension test. Comparison with the results of reference [10]

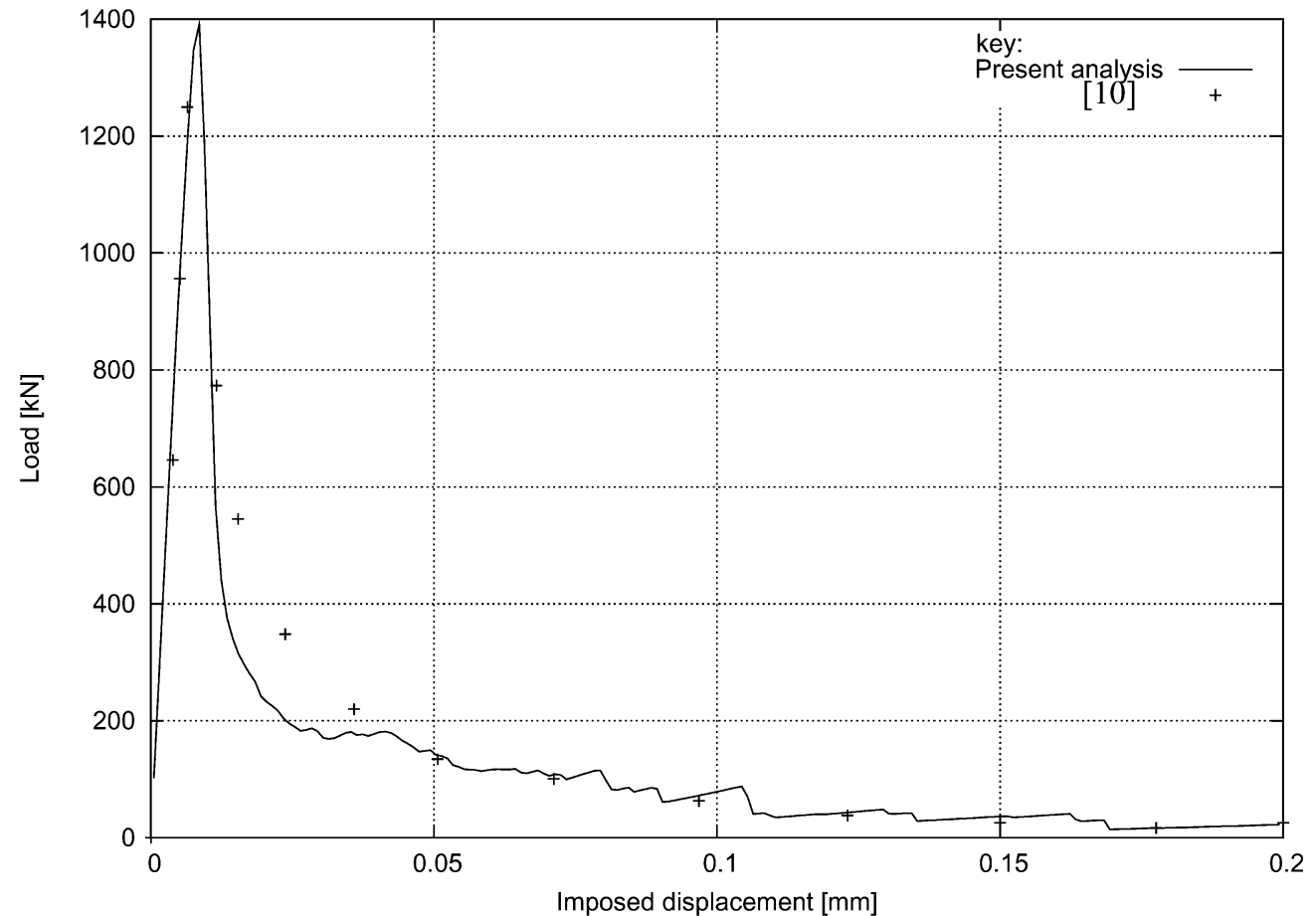




\subsection{Double edge-notched tension test}

The purpose of this test is to verify the robustness of the formulation for simultaneous growth of two cracks in mode I. Studies of this problem were accomplished in references $[10,46]$. A non-structured mesh is employed and the $15 \mathrm{~mm}$ notch offset is tested. In contrast with the procedures adopted in the above references, initial cracks are not present. For this particular notch offset, the cracks do not intersect.

The geometry, boundary conditions and relevant material properties are shown in Fig. (18).

The crack paths are shown in Fig. 19 for an imposed displacement value of $0.2 \mathrm{~mm}$. The initial crack tip locations are also not imposed, but are a result of the calculations.

It is interesting to verify that if the the crack closure effect is not accounted for, large interpenetration occurs in the right side of the mesh, near the crack tip. Fig. 20 shows both results, without closure enforcement and with closure enforcement.

The load-displacement curve comparison with the experimental results shown in reference [10] is presented in Fig. 21.

\section{Concluding remarks}

The proposed derivations allow very good results for $2 D$ brittle fracture, as was observed. It was shown that the KOS formulation can be dramatically improved by the incorporation of rotation variables and the new stress enhancement technique. The use of the enhanced strain technique was also found to be successful, specially for the coarse mesh analysed in the finite deformation case (the debonding example).

The verified solution robustness is excellent, which is a consequence of the exact linearization but also of the symmetric and isoparametric treatment of the rigid body displacement field induced by the crack.

Although the actual material model is still very simple, no restrictions are expected regarding the underlying material behavior.

Acknowledgements The funding provided by Ministrio da Ciência e Tecnologia e do Ensino Superior- Fundação para a Ciência e Tecnologia (Portugal) under the project POCTI/EME/41572/2001 is gratefully acknowledged. The first author acknowledges the helpful discussions with Dr. Esteban Samaniego and also with Prof. Pedro P. Camanho

\section{References}

1. Ortiz M, Leroy Y, Needlemann A (1987) A finite element method for localized failure analysis. Comput Meth Appl Mech Eng 61(2):189-214

2. Oliver J (1995) Continuum modelling of strong discontinuities in solid mechanics using damage models. Comput Mech 17:49-61
3. de Borst R, Wells GN, Sluys LJ (2001) Some observations on embedded discontinuity models. Eng Comput 18:241254

4. Bolzon G (2001) Formulation of a triangular finite element with an embedded interface via isoparametric mapping. Comput Mech 27:463-473

5. Jirasek M (2001) Comparative study on finite elements with embedded discontinuities Comput Meth Appl Mech Eng 188:307-330

6. Oliver J (1996) Modelling strong discontinuities in solid mechanics via strain softening constitutive equations. Part 1: Fundamentals Int J Numerical Meth Eng 39:3575-3600

7. Sluys LJ, Berends AH (1998) Discontinuous failure analysis for mode-I and mode-II localization problems Int $\mathbf{J}$ Solids Structures 35:4257-4274

8. Jirasek M, Zimmermann T (2001) Embedded crack model. I: Basic formulation Int J Numerical Meth Eng 50:12691290

9. Jirasek M, Zimmermann T (2001) Embedded crack model. II: Combination with smeared cracks Int J Numerical Meth Eng 50:1291-1305

10. Alfaiate J, Wells GN, Sluys LJ (2001) On the use of embedded discontinuity elements with crack path continuity for mode-I and mixed-mode fracture. Eng Fracture Mech 69:661-686

11. Alfaiate J, Simone A, Sluys LJ (2003) In: Bicanic N, de Borst R, Mang H, Meschke G (eds) A new approach to strong embedded discontinuities computational modelling of concrete structures EURO-C 2003 St. Johann im Pongau Salzburger Land, Austria

12. Oliver J, Huespe A, Pulido D, Samaniego E (2001) On the strong discontinuity approach in finite deformation settings. In: International Center Numerical Methods in Engineering 2001 Monograph CIMNE 62, Barcelona

13. Wells GN, de Borst R, Sluys LJ (2002) A consistent geometrically non-linear approach for delamination. Int J Numerical Meth Eng 54:1333-1355

14. Oliver J, Huespe AE, Pulido MDG, Samaniego E (2003) On the strong discontinuity approach in finite deformation settings. Int J Numerical Meth Eng 56:1051-1082

15. Moes N, Bolbow J, Belytschko T (1999) A finite element method for crack growth without remeshing. Int. J Numerical Meth Eng 46:131-150

16. Belytschko T, Chen H, Xu J, Zi G (2003) Dynamic crack propagation based on loss of hyperbolicity and a new discontinuous enrichment. Int $\mathbf{J}$ Numerical Meth Eng 58:1873-1905

17. Remmers JJC, de Borst R, Needleman A (2003) A cohesive segments method for the simulation of crack growth. Comput Mech 31:69-77

18. Oliver J, Huespe AE, Samaniego E, (2003) A study on finite elements for capturing strong discontinuities. Int J Numerical Meth Eng 56:2135-2161

19. Rousselier G, Devaux J-C, Mottet G, Devesa G, Landes JD, Saxena A, Merkle JG (1989) Nonlinear fracture mechanics: volume II-Elastic-plastic fracture "A methodology for ductile fracture analysis based on damage mechanics: an illustration of a local approach of fracture. American Society for Testing and Materials 1989 STP 995, Philadelphia pp 332-354

20. de Borst R, Pamin J, Geers MGD (1999) On coupled gradient-dependent plasticity and damage theories with a view to localization analysis. Eur J Mech A/Solids 18:939 962

21. Kim JH, Kim SJ, Kim WD (1999) Numerical simulation of ductile fracture based on local approach concept ASCE. J Eng Mech 125 8:975-978 
22. Peerlings RHJ, Brekelmans WAM, de Borst R, Geers MGD (2001) Mathematical and numerical aspects of an elasticity-based local approach to fracture. Revue Europeenne des Elements Finis 10:209-226

23. de Borst R (2001) Some recent issues in computational failure mechanics. Int J Numerical Meth Eng 52:63-95

24. Areias PMA, César de Sá JMA, Conceição António CA (2002) Finite strain plasticity including softening: a gradient damage model, In: Mang HA, Rammerstorfer FG, Eberhardsteiner $\mathbf{J}$ (eds) Proceedings of the Fifth World Congress on Computational Mechanics (WCCM V) Vienna University of Technology, Austria. http:// wccm.tuwien.ac.at July 7-12 ISBN 3-9501554-0-6

25. Lofti HR, Shing PB (1995) Embedded representation of fracture in concrete with mixed finite elements. Int J Numerical Meth Eng 38:1307-1325

26. Areias PMA, César de Sá JMA, Conceição António CA, (2003) Strong displacement discontinuities and Lagrange multipliers: finite displacement formulations in the analysis of fracture problems. Recent developments in the modelling of rupture in solids pp, 105-110 Foz do Iguau Brasil 47 August 2003

27. Wells GN, Sluys LJ (2001) A new method for modelling cohesive cracks using finite elements. Int $\mathbf{J}$ Numer Meth Eng 50:2667-2682

28. Simo JC, Rifai MS (1990) A class of mixed assumed strain methods and the method of incompatible modes. Int $\mathbf{J}$ Numerical Meth Eng 29:1595-1638

29. Simo JC, Armero F (1992) Geometrically non-linear enhanced strain mixed methods and the method of incompatible modes. Int J Numerical Meth Eng 33:1413-1449

30. Areias PMA, César de Sá JMA, Conceição António CA, Fernandes AA (2003) Analysis of 3D problems using a new enhanced strain hexahedral element. Int. J. Numer. Meth. Eng. 58:1637-1682

31. Haupt P (2002) Continuum Mechanics and Theory of Materials Springer-Verlag Second

32. César de Sá JMA, Areias PMA, Natal Jorge RM (2001) Quadrilateral elements for the solution of elasto-plastic finite strain problems. Int J Numerical Meth Eng 51:883-917

33. Oliver J (2000) On the discrete constitutive models induced by strong discontinuity kinematics and continuum constitutive equations. Int J Solids Structures 37:7207-7229
34. Oliver J, Huespe AE, Pulido MDG, Chaves E (2002) From continuum mechanics to fracture mechanics: the strong discontinuity approach. Eng Fracture Mech 69:113-136

35. Mariani S, Perego U (2003) Extended finite element method for quasi-brittle fracture Int J Numerical Meth Eng 58:103-126

36. Elices M, Guinea GV, Gómez J, Planas J (2002) The cohesive zone model: advantages limitations and challenges. Eng Fracture Mech 69:137-163

37. Camanho PP, Davila CG (2002) Mixed-mode decohesion finite elements for the simulation of delamination in composite materials NASA TM-2002-211737 Hampton Virginia 23681-2199

38. de Borst R (2002) Fracture in quasi-brittle materials: a review of continuum damage-based approaches. Eng Fracture Mech 69:95-112

39. Boussa D, Aravas N (2001) A numeric-symbolic approach to the problem of localization of plastic flow. Comput Mech 27:324-331

40. Oliver J (1996) Modelling strong discontinuities in solid mechanics via strain softening constitutive equations. Part 1: Numerical simulation. Int $\mathbf{J}$ Numerical Meth Eng 39:3601-3623

41. Hao S, Liu WK, Qian D, (2000) Localization-induced band and cohesive model. ASME J Appl Mech 67:803-812

42. Siegmund T, Brocks W (2000) A numerical study on the correlation between the work of separation and the dissipation rate in ductile fracture. Eng Fracture Mech 67:139-154

43. Comi C, Driemeier L, de Borst R, van der Giessen E (1998) Material Instabilities in Solids 26 John Wiley and Sons 425-440

44. Zi G, Belytschko T (2003) New crack-tip elements for XFEM and applications to cohesive cracks. Int J Numerical Meth Eng 57:2221-2240

45. Galvez JC, Elices M, Guinea GV, Planas J (1998) Mixed mode fracture of concrete under proportional and nonproportional loading. Int J Fracture 94:267-284

46. Wang TK, Chang CS, van Mier JGM, Sluys LJ Bittencourt TN (2001) Fracture modeling of concrete using two different microstructural mechanics concepts 2001 Mechanics and Materials Summer Conference 2001. University of California, San Diego ASME ASCE SES 